\title{
Low-frequency Landau-Zener-Stückelberg interference in dissipative superconducting qubits
}

\author{
Lingjie $\mathrm{Du}^{1,2}$, Dong $\mathrm{Lan}^{1}$, and Yang $\mathrm{Yu}^{1}$ \\ ${ }^{1}$ National Laboratory of Solid State Microstructures and Department of Physics, Nanjing University \\ Nanjing 210093, China \\ ${ }^{2}$ Department of Physics and Astronomy, Rice University, Houston, Texas 77251-1892, USA \\ E-mail: lingjie.du@rice.edu \\ yuyang@nju.edu.cn
}

Received January 22, 2013, revised April 3, 2013

\begin{abstract}
Landau-Zener-Stückelberg (LZS) interference of continuously driven superconducting qubits is studied. Going beyond the second order perturbation expansion, we find a time dependent stationary population evolution as well as unsymmetrical microwave driven Landau-Zener transitions, resulting from the nonresonant terms which are neglected in rotating-wave approximation. For the low-frequency driving, the qubit population at equilibrium is a periodical function of time, owing to the contribution of the nonresonant terms. In order to obtain the average population, it is found that the average approximation based on the perturbation approach can be applied to the low-frequency region. For the extremely low frequency which is much smaller than the decoherence rate, we develop noncoherence approximation by dividing the evolution into discrete time steps during which the coherence is lost totally. These approximations present comprehensive analytical descriptions of LZS interference in most of parameter space of frequency and decoherence rate, agreeing well with those of the numerical simulations and providing a simple but integrated understanding to system dynamics. The application of our models to microwave cooling can obtain the minimal frequency to realize effective microwave cooling.
\end{abstract}

PACS: 03.65.Yz Decoherence; open systems; quantum statistical methods;

85.25.Dq Superconducting quantum interference devices (SQUIDs);

85.25.Cp Josephson devices;

03.67.-a Quantum information.

Keywords: superconducting qubits, population evolution, effective microwave cooling.

\section{Introduction}

Superconducting devises based on Josephson tunnel junctions have great importance in demonstrating quantum phenomena at macroscopic scales and hold promise for applications in quantum information [1-5]. In the studies of these systems, crossovers between energy levels are usually associated with a wide variety of interesting phenomena, particularly in connection with Landau-ZenerStückelberg (LZS) interference [6-8].

Recently, a series of great progresses [9-20] have been implemented to study the coherent dynamics of qubits in the regime dominated by LZS interference. The strong high-frequency microwave generates controllable population transitions, allowing for fast and reliable control of quantum systems. The delicate LZS interference patterns also provide a way to calibrate the parameters of the qubit and its interaction with the environment. On the other hand, through continuous Landau-Zener (LZ) transitions, the low-frequency microwave can be used to achieve an effective temperature of qubit up to two orders of magnitude lower than the bath temperature through an analogue of optical sideband cooling [21,22]. This microwave cooling provides an effective means for preparing qubit state with high fidelity and suppressing decoherence. Therefore, understanding dynamics of LZS interference in strongly driven superconducting qubits has both fundamental and practical significances. A number of theoretical investigations [6,14,23-41] have been developed in driven quantum two-level systems (TLS). These works generally addressed the coherent phenomena in the high-frequency driving in superconducting qubits, as reviewed recently in Ref. 6. In the realistic situation, the influence of environment always needs to be considered. Compared with natural atoms, the solid state systems contain macroscopic degrees of freedom strongly coupled to environment bathes that decohere the quantum states to be controlled. To address the driven 
dissipative TLS, two typical methods usually taken into account are rotating-wave approximation (RWA) [32,41] and perturbation-RWA [19,27].

RWA provides a simple physical picture and good approximation for the stationary population. However, it limits at the high-frequency region, i.e. $\omega \gg \Gamma_{2}, \Delta J_{n}(A / \omega)$, where $n$ is the closest integer to $\varepsilon_{0} / \omega$ and $\varepsilon_{0}$ is the dc detuning. The other method, perturbation-RWA, applies the second order perturbation approximation based on RWA and can be used in the parameter region with the high frequency and strong decoherence, i.e. $\omega>\Delta, \Gamma_{2}$ and $\Gamma_{2} \gg W_{01}$, where $W_{01}$ is microwave driven LandauZener (MDLZ) transition rate from $|0\rangle$ and $|1\rangle$. However in the low-frequency driving where microwave cooling takes effects, both methods would be inappropriate.

In this article, we focus on analyzing the stationary state which is useful in spectroscopy experiments $[9,10,12,15]$ and microwave cooling [21] in the presence of decoherence. Going beyond the second order perturbation and focusing on the nonresonant terms which are dropped in RWA, we present the real-time population evolution as well as unsymmetrical MDLZ transitions in a strongly driven TLS of the long-time dynamics. In the high-frequency case, i.e. $\omega>\Delta$, the result of perturbation approach can fully cover those of RWA and perturbation-RWA. In addition, the perturbation approach can be applied to larger parameters space, at which other methods are inappropriate.

Then we move on lower frequency region, i.e. $\omega<\Delta$. In this parameter region, microwave cooling begins to take effects, motivating us to give a thorough understanding of the dynamics. The steady dynamics shows a periodic time dependence of qubit population, which is from the nonresonant terms and cannot be explained by perturbation-RWA. Then we focus on the average stationary population. Although one can use the perturbation approach, it is cumbersome thus difficult to get the final solutions. Therefore, we use the average perturbation approximation (APA), which is based on perturbation approach through the average over one driving period. Interestingly, the analytical expression in APA has the same form with that of perturbation-RWA. If the frequency further decreases, APA cannot correctly give the qubit population. We thereby propose the noncoherence approximation (NCA). The incoherent evolution is divided into discrete incoherent time steps, from which we can obtain the analytical solution. It is found that when the frequency is much smaller than the decoherence rate, the time dependent and average population calculated by NCA agree quantitatively with those of the numerical simulation. At last, we extend the analytical results of APA and NCA to the multi-level system. We discuss microwave cooling [21] and obtain the minimal frequency of the effective microwave cooling.

Through these approximations of perturbation approach, we present simple and comprehensive descriptions in the average stationary population in most of parameter

space of frequency and decoherence rate. The agreement with numerical results indicates that the picture captures the underlying physics, thus giving a better understanding of the behavior of system dynamics. The models and methods we used here are also valid for other TLSs with similar structures.

The article is organized as follows. In Sec. 2, we introduce the basic model and approximations. In Sec. 3, assuming high order perturbation in the long-time dynamics, we present the perturbation approach which considers the nonresonant terms. Then with this approach we discuss the high-frequency driving case. In Sec. 4, we discuss the average stationary population in the low frequency. Through APA, we obtain the average population in the steady case under the low-frequency driving. In Sec. 5, for the extremely low frequency, we demonstrate that NCA can be used to get the analytical solution, which is useful to describe the system dynamics. In Sec. 6, we extend our model to multi-level flux qubit and discuss the frequency range of effective microwave cooling.

\section{Basic model}

For a superconducting flux qubit [42,43] with three Josephson tunnel junctions, if the external flux bias $\Phi \approx 0.5 \Phi_{0}$, where $\Phi_{0}=h / 2 e$ is the flux quantum, a double-well landscape of the potential energy parameterized by the dc flux detuning $\delta \Phi_{d c}=\Phi-0.5 \Phi_{0}$ exists as shown in Fig. 1. In the millikelvin temperature $\sim 10 \mathrm{mK}$, a series of diabatic states are localized in different wells. States $|0\rangle$ and $|1\rangle$ are the lowest levels in the left and right wells, respectively. They are characterized by different flowing directions of the persistent currents, namely clockwise and anticlockwise. The states in each well are separated by large energy spacing on the order of $20 \mathrm{GHz}$ [15], while the coupling strength between two wells is small compared with the energy spacing. As the qubit is driven with a microwave $\Phi_{a c}=\Phi_{r f} \sin \omega t$, where $\Phi_{r f}$ is the microwave amplitude, the time dependent flux detuning is

$$
\delta \Phi(t)=\delta \Phi_{d c}+\Phi_{a c}=\delta \Phi_{d c}+\Phi_{r f} \sin \omega t .
$$

(a)

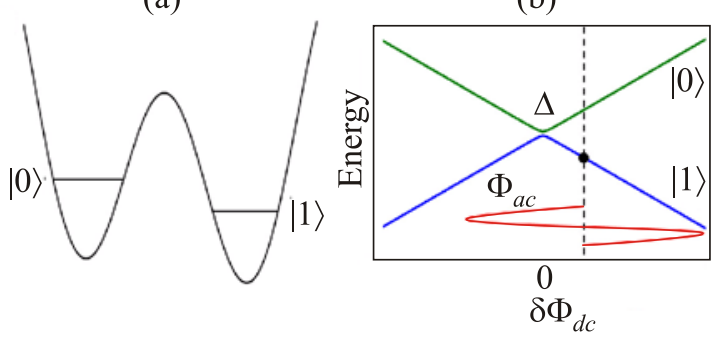

Fig. 1. (Color online) (a) Schematic picture of the double-well potential of a superconducting flux qubit. States $|1\rangle$ is in the right well; $|0\rangle$ is in the left well. (b) Schematic energy diagram of the flux qubit. Red solid curve represents the microwave $\Phi_{r f} \sin \omega t$. The vertical dashed line marks a particular static flux detuning $\delta \Phi_{d c}$. 
Then the time dependent energy detuning of states $|0\rangle$ and |1) can be described as

$$
\varepsilon(t)=\varepsilon_{0}+A \sin \omega t,
$$

where $\varepsilon_{0}=\left(\left|m_{0}\right|+\left|m_{1}\right|\right) \delta \Phi_{d c}$ is the dc energy detuning of states $|0\rangle$ and $|1\rangle, \quad A=\left(\left|m_{0}\right|+\left|m_{1}\right|\right) \Phi_{r f}$ is the microwave energy amplitude, and $m_{i}=d E_{i}(\Phi) / d \Phi$ is the diabatic energy-level slope of state $|i\rangle$ in units of frequency per flux. For the initial time $t=0$, the qubit is at the detuning $\varepsilon(0)=\varepsilon_{0}$.

We start from a periodically driven TLS coupled to an environment bath. Such a process can be described by Hamiltonian (we set $\hbar=k_{B}=1$ ) [26,27,40,44,45]

$$
H=-\frac{\Delta}{2} \sigma_{X}-\frac{\varepsilon(t)}{2} \sigma_{z}+H_{\mathrm{int}}+H_{B} \text {, }
$$

where $\sigma_{X}$ and $\sigma_{z}$ are Pauli matrices, $\sigma_{z}=|1\rangle\langle 1|-| 0\rangle\langle 0|$ and $\sigma_{X}=|1\rangle\langle 0|+| 0\rangle\langle 1|, \quad H_{\text {int }}$ is the system-environment interaction Hamiltonian, and $H_{B}$ is the environment Hamiltonian. We assume the qubit is embedded in the bath in equilibrium at temperature $T$ with the density matrix of environment $\rho_{B}=\mathrm{e}^{-H_{B} / T}$. The system-environment interaction Hamiltonian is written as

$$
H_{\text {int }}=-Q \sigma_{Z} / 2 \text {, }
$$

where $Q$ is an operator acting on the environment.

Before investigating the system dynamics in the presence of the environment bath, it is worth recalling main features of its free time evolution which is described by Hamiltonian Eq. (1) without $H_{\text {int }}$ and $H_{B}$. By a gauge transformation, the Hamiltonian is brought to the form

$$
H_{1}=-\frac{\Delta}{2} \mathrm{e}^{\phi(t)}|1\rangle\langle 0|+\text { H.c. },
$$

where

$$
\phi(t)=-i \int_{0}^{t} \varepsilon(\tau) d \tau
$$

Using Bessel functions, we have

$$
\mathrm{e}^{\phi(t)}=\mathrm{e}^{-i \varepsilon_{0} t-i A / \omega} \sum J_{n}\left(\frac{A}{\omega}\right) \mathrm{e}^{i n(\omega t+\pi / 2)}
$$

and find

$$
H_{2}=-\frac{\Delta}{2} \sum J_{n}\left(\frac{A}{\omega}\right) \mathrm{e}^{-i\left(\varepsilon_{0} t-n \omega t\right)+i n \pi / 2-i A / \omega}|1\rangle\langle 0|+\text { H.c. }
$$

With the relation $\mathrm{e}^{i A / \omega}=\sum J_{n}(A / \omega) \mathrm{e}^{i n \pi / 2}$, we consider parameters close to the $n$-photon resonance, where $n \omega=\varepsilon_{0}$ and rely on RWA to neglect the fast oscillating terms with $k \neq n$ for the high frequency $\omega$. Therefore, when the system is initially in state $|1\rangle$ we have the population in state $|0\rangle[6,9,32]$

$$
\begin{aligned}
& \rho_{00}=\frac{1}{2} \sum \frac{\Delta^{2} J_{n}^{2}(A / \omega)}{\Delta^{2} J_{n}^{2}(A / \omega)+\left(\varepsilon_{0}-n \omega\right)^{2}} \times \\
& \times\left[1-\cos \sqrt{\Delta^{2} J_{n}^{2}(A / \omega)+\left(\varepsilon_{0}-n \omega\right)^{2}} t\right] .
\end{aligned}
$$

This approximation neglects all the nonresonant terms which oscillate very fast in the time scale of system dynamics, so the high frequency is needed to keep the validity of RWA, i.e. $\omega>\Delta \gg \Delta J_{n}(A / \omega)[6,29,32]$. We compare the real-time numerical solution with the analytical results in Eq. (5), shown in Fig. 2. RWA has the high accuracy under the high-frequency driving. However, when the frequency is low, i.e. $\omega<\Delta$, the difference between numerical and RWA results are not negligible. It should be noted that in Fig. 2(a) there exist two kinds of oscillations. One agrees with Eq. (5) while another as shown in the panel in Fig. 2(a) is from the nonresonant terms in Eq. (4). When the frequency is small just as shown in Fig. 2(b), the effect of nonresonant terms could not be neglected. In next sections, we will further show the influence of these nonresonant terms on system dynamics.

In the realistic solid-state system, the decoherence which is always strong needs to be taken into account. In this case, the Bloch equations are usually used to provide the numerical stimulation, which read

$$
\dot{\rho}=-i\left[H_{1}, \rho\right]+\Gamma[\rho],
$$

where $\Gamma[\rho]$ is the dissipative term $[46,47]$

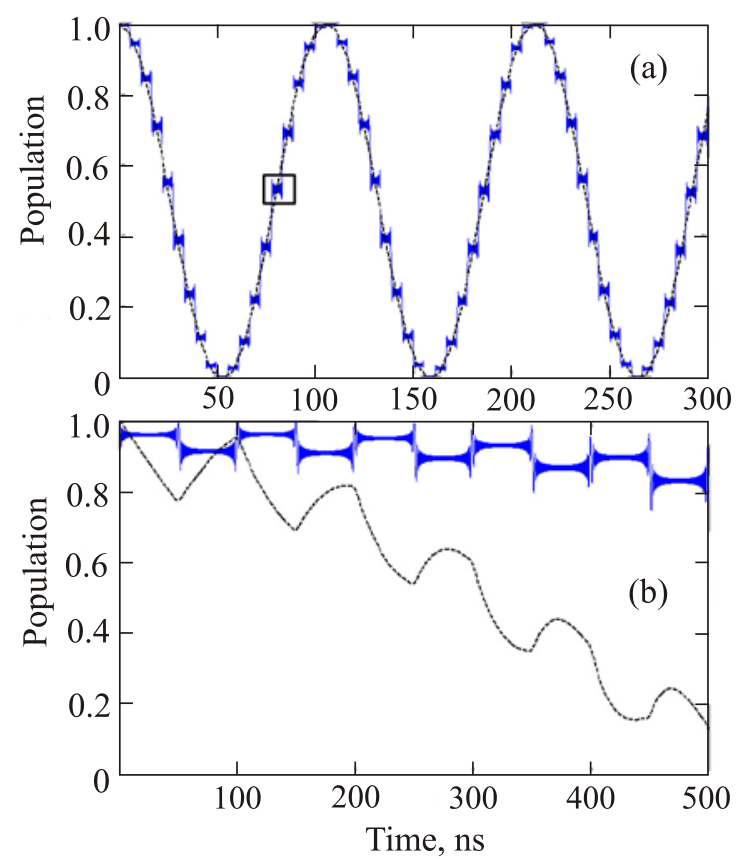

Fig. 2. (Color online) Population in state $|1\rangle$ as a function of time, assuming that the system is initially in state $|1\rangle$. In both figures, $\varepsilon_{0} / 2 \pi=90 \mathrm{MHz}, \Delta / 2 \pi=90 \mathrm{MHz}$, and $A / 2 \pi=5 \mathrm{GHz}$. The microwave frequency is $\omega / 2 \pi=90 \mathrm{MHz}$ (a) and $10 \mathrm{MHz}$ (b), respectively. The blue solid line shows the numerical stimulations and the black dotted line shows the RWA results. 


$$
\Gamma[\rho]=\left[\begin{array}{cc}
-\Gamma_{10} \rho_{11}+\Gamma_{01} \rho_{00} & -\Gamma_{2} \rho_{01} \\
-\Gamma_{2} \rho_{10} & \Gamma_{10} \rho_{11}-\Gamma_{01} \rho_{00}
\end{array}\right],
$$

where $\Gamma_{2}=1 / T_{2}$ is the decoherence rate, $T_{2}$ is the decoherence time, $\Gamma_{i j}$ is the interwell relaxation rate from state $|i\rangle$ to $|j\rangle, \quad \Gamma_{10}=\Gamma_{01} \mathrm{e}^{-\varepsilon_{0} / T}, \quad 1 / T_{1}=\max \left\{\Gamma_{10}, \Gamma_{01}\right\}$, and $T_{1}$ is the interwell relaxation time. After the long-time system dynamical evolution, whatever the initial state is, the temporal oscillation in Fig. 2 is eliminated by the decoherence and the qubit population will converge to a stationary solution. We make RWA with the Eq. (6), neglect the fast oscillating terms, and obtain the probability in state $|0\rangle[6]$

$$
\rho_{00}=\sum_{n} \frac{\frac{\Delta^{2} J_{n}^{2}(A / \omega) \Gamma_{2}}{2\left[\Gamma_{2}^{2}+\left(\varepsilon_{0}-n \omega\right)^{2}\right]}+\Gamma_{10}}{\frac{\Delta^{2} J_{n}^{2}(A / \omega) \Gamma_{2}}{\Gamma_{2}^{2}+\left(\varepsilon_{0}-n \omega\right)^{2}}+\Gamma_{01}+\Gamma_{10}} .
$$

To ensure Eq. (8) is valid, we need not only the high frequency, i.e., $\omega>\Delta \gg \Delta J_{n}(A / \omega)$, but also the high coherence, i.e., $\omega \gg \Gamma_{2}$. If the decoherence is strong $\omega \sim \Gamma_{2}$, the population $\rho_{00}$ may even be larger than 1 and this stationary solution is obviously not reasonable.

In order to describe the dynamics in the strong decoherence, Berns et al. [19] proposed the perturbationrotating-wave approximation. Under the Gaussian white low-frequency noise, based on the second order expansion perturbation, we have MDLZ transition rate from state $|0\rangle$ to $|1\rangle$

$$
W_{01}=\frac{\Delta^{2}}{2} \sum_{n} \frac{\Gamma_{2} J_{n}^{2}(A / \omega)}{\left(\varepsilon_{0}-n \omega\right)^{2}+\Gamma_{2}^{2}} .
$$

Correspondingly, $W_{10}\left(\varepsilon_{0}\right)=W_{01}\left(-\varepsilon_{0}\right)=W_{01}\left(\varepsilon_{0}\right)$. Then for the long-time dynamics, we employ the rate equations, in which the qubit population in state $|0\rangle$ and $|1\rangle$, i.e., $\rho_{00}$ and $\rho_{11}$, follow

$$
\begin{gathered}
\dot{\rho}_{00}=-\left(W_{01}+\Gamma_{01}\right) \rho_{00}+\left(W_{10}+\Gamma_{10}\right) \rho_{11}, \\
1=\rho_{00}+\rho_{11} .
\end{gathered}
$$

Since MDLZ transition rate is constant in the stationary case, the equations would lead to the constant population, i.e. $\dot{\rho}_{00}=\dot{\rho}_{11}=0$, and the population in state $|0\rangle$ becomes

$$
\rho_{00}=\sum_{n} \frac{\frac{\Delta^{2} J_{n}^{2}(A / \omega) \Gamma_{2}}{2\left[\Gamma_{2}^{2}+\left(\varepsilon_{0}-n \omega\right)^{2}\right]}+\Gamma_{10}}{\sum_{n} \frac{\Delta^{2} J_{n}^{2}(A / \omega) \Gamma_{2}}{\Gamma_{2}^{2}+\left(\varepsilon_{0}-n \omega\right)^{2}}+\Gamma_{01}+\Gamma_{10}} .
$$

In order to ensure the validity of this approach [19,27], the high frequency and strong decoherence, i.e., $\omega>\Delta, \Gamma_{2}$ and $\Gamma_{2} \gg W_{01}$, are necessary, which greatly restrict the application of this approximation. In next sections, we will introduce approximations to extend the quantitative analysis to larger parameters space.

\section{Perturbation approach}

In order to get analytical results in larger parameters space, we go back to Hamiltonian (1) and take the perturbation approach. First of all, we follow the process in Refs. 27 and 44, and the complete time evolution operator in the laboratory picture can be expressed as

$$
\begin{gathered}
U\left(t_{1}, t_{2}\right)=\mathrm{e}^{-i H_{B} t_{1}} U_{0}\left(t_{1}\right) U_{1}\left(t_{1}\right) U_{V}\left(t_{1}, t_{2}\right) \times \\
\times U_{1}^{\dagger}\left(t_{2}\right) U_{0}^{\dagger}\left(t_{2}\right) \mathrm{e}^{i H_{B} t_{2}},
\end{gathered}
$$

where

$$
\begin{aligned}
& U_{0}(t)=\mathcal{T} \exp \left[\frac{i}{2} \int_{0}^{t} d \tau \varepsilon(\tau) \sigma_{z}\right], \\
& U_{1}(t)=\mathcal{T} \exp \left[\frac{i}{2} \int_{0}^{t} d \tau Q(\tau) \sigma_{z}\right], \\
& Q(t)=\mathrm{e}^{i H_{B} t} Q \mathrm{e}^{-i H_{B} t}, \quad U_{V}\left(t_{1}, t_{2}\right)=\mathcal{T} \exp \left[-i \int_{t_{2}}^{t_{1}} d \tau V(\tau)\right], \\
& V(t)=-\frac{\Delta}{2} \sum_{n} J_{n}\left(\frac{A}{\omega}\right) \exp \left[-i\left(\varepsilon_{0} t-n \omega t\right)+\right. \\
& \left.+\frac{i n \pi}{2}-i \frac{A}{\omega}\right] U_{1}^{\dagger}(t)|1\rangle\langle 0| U_{1}(t)+\text { H.c. },
\end{aligned}
$$

and $\mathcal{T}$ is the time ordering operator. Let $\rho_{S B}$ express the total density matrix of the system plus environment bath, which can be described as $\rho_{S B}(t)=U(t, 0) \rho_{S B}(0) U^{\dagger}(t, 0)$. Then the system reduced density matrix is defined as $\rho(t)=\operatorname{Tr}_{B}\left\{\rho_{S B}(t)\right\}$, where $\operatorname{Tr}_{B}\{\ldots\}$ denotes the trace over the environmental degrees of freedom. We assume at the initial time the density matrix is separable, i.e., $\rho_{S B}(0)=\rho(0) \otimes \rho_{B}$, and the system is initialized in state $|0\rangle$, i.e., $\rho(0)=|0\rangle\langle 0|$.

We perform a perturbation expansion of $U_{V}$ in $V(\tau)$, which reads

$$
\begin{gathered}
U_{V}(t, 0) \approx 1-i \int_{0}^{t} d \tau V(\tau)-\int_{0}^{t} d \tau V(\tau) \int_{0}^{\tau} d \tau^{\prime} V\left(\tau^{\prime}\right)+ \\
+i \int_{0}^{t} d \tau V(\tau) \int_{0}^{\tau} d \tau^{\prime} V\left(\tau^{\prime}\right) \int_{0}^{\tau^{\prime}} d \tau^{\prime \prime} V\left(\tau^{\prime \prime}\right)+\ldots
\end{gathered}
$$

For the strong decoherence, i.e., $\Gamma_{2} \gg \Delta J_{n}(A / \omega) \sim W_{01}$, we can consider the expansion to the second order in a time interval $1 / \Gamma_{2} \ll t<1 /\left[\Delta J_{n}(A / \omega)\right][27,45]$. However, in the high-coherence region where $\Gamma_{2}<\Delta J_{n}(A / \omega)$, instead of considering the time interval $t<1 /\left[\Delta J_{n}(A / \omega)\right]$, we need take account of a time interval $t$ much larger than the decoherence time. Hence this time interval $t$ is also much larger than $1 /\left[\Delta J_{n}(A / \omega)\right]$. Moreover, for the low frequency 
$\Delta J_{n}(A / \omega) \lesssim \omega<\Delta$, in order to calculate MDLZ transition in the stationary case, even if $1 /\left[\Delta J_{n}(A / \omega)\right]$ is much longer than the decoherence time, the time interval $t<1 /\left[\Delta J_{n}(A / \omega)\right]$ is still not sufficiently long relative to the driving period. As a result we consider $t \gg 1 / \omega$,
$1 /\left[\Delta J_{n}(A / \omega)\right], 1 / \Gamma_{2}$. It is clear that the second order perturbation expansion would be insufficient $[27,45]$. Considering high order expansion terms, we obtain the population in state $|1\rangle$ (see Appendix)

$$
\begin{gathered}
\rho_{11}(t) \approx \int_{0}^{t} \int_{0}^{t} d \tau d \tau^{\prime} \operatorname{Tr}_{B}\left\{\left\langle 1\left|V(\tau) \rho_{S B}(0) V^{\dagger}\left(\tau^{\prime}\right)\right| 1\right\rangle\right\}-2 \int_{0}^{t} \int_{0}^{t} d t_{1} d t_{2} \operatorname{Tr}_{B}\left\{\left\langle 1\left|\left[\int_{0}^{t} \int_{0}^{t} d t_{3} d t_{4} V\left(t_{4}\right) \rho_{S B}(0) V^{\dagger}\left(t_{3}\right)\right] V^{\dagger}\left(t_{2}\right) V^{\dagger}\left(t_{1}\right)\right| 1\right\rangle\right\}- \\
-\operatorname{Tr}_{B}\left\{\int_{0}^{t} \int_{0}^{t} d t_{1} d t_{4}\left\langle 1\left|V\left(t_{4}\right)\left[2 \int_{0}^{t} \int_{0}^{t} d t_{2} d t_{3} \rho_{S B}(0) V^{\dagger}\left(t_{3}\right) V^{\dagger}\left(t_{2}\right)\right] V^{\dagger}\left(t_{1}\right)\right| 1\right\rangle\right\}+\ldots
\end{gathered}
$$

For the short time interval, i.e., $t<1 /\left[\Delta J_{n}(A / \omega)\right]$, we use the second order perturbation expansion and can keep only the first term in Eq. (14) expressing the population in state $|1\rangle$ transferred from $|0\rangle$, just as discussed in Ref. 27. As the time goes on, the population is not completely in state $|0\rangle$. The first term does not consider the effect of the reduced population in state $|0\rangle$ such that it cannot characterize the population transferred from state $|0\rangle$ to $|1\rangle$ any more. Higher order terms in $U_{V}(t, 0)$ need to be considered, which result into the second as well as third term in Eq. (14). In the second term,

$$
\int_{0}^{t} \int_{0}^{t} d t_{3} d t_{4} V\left(t_{4}\right) \rho_{S B}(0) V^{\dagger}\left(t_{3}\right)
$$

describes the population transferred from state $|0\rangle$ to $|1\rangle$ in the time interval $t$. Then the second term characterizes the transition of population from state $|1\rangle$ to $|0\rangle$ based on this part of population in $|1\rangle$. Similarly,

$$
2 \int_{0}^{t} \int_{0}^{t_{2}} d t_{2} d t_{3} \rho_{S B}(0) V^{\dagger}\left(t_{3}\right) V^{\dagger}\left(t_{2}\right)
$$

in the third term describes the reduced population in state $|0\rangle$ in the time interval $t$. The third term describes the decrease of population transferred from state $|0\rangle$ to $|1\rangle$ because of the reduced population in state $|0\rangle$. When the time interval $t$ becomes further larger, more terms will appear in Eq. (14) as a ripple effect and we can also extend the integration limit $t$ to $\infty$.

Then we only discuss the first term in Eq. (14) as an example, and other terms can be treated with a similar procedure. We rewrite the first term of Eq. (14) to

$$
\begin{aligned}
& y(t)=\frac{\Delta^{2}}{4} \int_{0}^{t} d t_{1} \int_{0}^{t} d t_{2} K\left\langle U_{-}^{+}\left(t_{1}\right) U_{+}\left(t_{1}\right) U_{+}^{+}\left(t_{2}\right) U_{-}\left(t_{2}\right)\right\rangle, \\
& K=\sum_{n, m} J_{n}\left(\frac{A}{\omega}\right) J_{m}\left(\frac{A}{\omega}\right) \mathrm{e}^{i\left(\varepsilon_{0}-m \omega\right) t_{1}-i\left(\varepsilon_{0}-n \omega\right) t_{2}+i n \pi / 2-i m \pi / 2},
\end{aligned}
$$

where

$$
U_{ \pm}(t)=\mathcal{T} \exp \left[ \pm(i / 2) \int_{0}^{t} Q(\tau) d \tau\right]
$$

For the low frequency, i.e., $\Delta J_{n}(A / \omega) \lesssim \omega<\Delta$, the terms in $K$ with $n \neq m$ would oscillate not fast enough, leading the invalidity of RWA. Assuming the white Gaussian low-frequency noise, we rewrite Eq. (15) to

$$
\begin{gathered}
y(t)=\frac{\Delta^{2}}{4} \int_{0}^{t} d t_{1} \int_{0}^{t} d t_{2} \sum_{m} \mathrm{e}^{i\left(\varepsilon_{0}-m \omega\right)\left(t_{1}-t_{2}\right)-\Gamma_{2}\left|t_{1}-t_{2}\right|} \times \\
\times\left\{J_{m}^{2}\left(\frac{A}{\omega}\right)+\sum_{n \neq 0} J_{n+m}\left(\frac{A}{\omega}\right) J_{m}\left(\frac{A}{\omega}\right) \mathrm{e}^{i n \omega t_{2}+i n \pi / 2}\right\} .
\end{gathered}
$$

For the long-time dynamics, we extend the integration limits of Eq. (16) to $\infty$, and have [27]

$$
\begin{gathered}
\dot{y}(t)=\frac{\Delta^{2}}{2} \sum_{n} \frac{\Gamma_{2} J_{n}^{2}(A / \omega)}{\left(\varepsilon_{0}-n \omega\right)^{2}+\Gamma_{2}^{2}}+f(t), \\
f(t)=\frac{\Delta^{2}}{4} \sum_{n \neq 0, m} \lim _{t \rightarrow \infty} \frac{d}{d t} \int_{0}^{t} d t_{1} \int_{0}^{t} d t_{2} J_{n+m}\left(\frac{A}{\omega}\right) J_{m}\left(\frac{A}{\omega}\right) \times \\
\times \mathrm{e}^{i\left(\varepsilon_{0}-m \omega\right) \tau+i n \omega t_{2}+i n \pi / 2-\Gamma_{2}|\tau|},
\end{gathered}
$$

where $\tau=t_{1}-t_{2}$. The first term in Eq. (17) is the familiar result in perturbation-RWA. For convenience we change the form of $f(t)$ to

$$
\begin{gathered}
f(t)=\frac{\Delta^{2}}{4} \sum_{n \neq 0, m} J_{n+m}\left(\frac{A}{\omega}\right) J_{m}\left(\frac{A}{\omega}\right) \times \\
\times \lim _{t \rightarrow \infty} \frac{d}{d t} \int_{0}^{t} d \tau^{\prime} \int_{-\tilde{t}}^{\tilde{t}} d \tau \mathrm{e}^{i\left(\varepsilon_{0}-m \omega\right) \tau+i n \omega\left(\tau^{\prime}-\tau / 2\right)+i n \pi / 2-\Gamma_{2}|\tau|},
\end{gathered}
$$

where $\tau^{\prime}=\left(t_{1}+t_{2}\right) / 2$ and $\tilde{t}=\min \left\{2 \tau^{\prime}, 2 t-2 \tau^{\prime}\right\}$. Therefore we have the leading term given by

$$
f(t) \approx \frac{\Delta^{2}}{2} \sum_{n \neq 0, m} J_{n+m}\left(\frac{A}{\omega}\right) J_{m}\left(\frac{A}{\omega}\right) \frac{\Gamma_{2} \cos n(\omega t+\pi / 2)}{\left(\varepsilon_{0}-m \omega\right)^{2}+\Gamma_{2}^{2}} .
$$


Then we consider other terms in Eq. (14) and follow the above process, obtaining

$$
\begin{gathered}
\dot{\rho}_{11}(t) \approx \dot{y}\left(t, \varepsilon_{0}\right)-\dot{y}\left(t, \varepsilon_{0}\right) \rho_{11}(t)-\dot{y}\left(t,-\varepsilon_{0}\right) \rho_{11}(t)= \\
=W_{01}(t) \rho_{00}(t)-W_{10}(t) \rho_{11}(t),
\end{gathered}
$$

where $W_{01}\left(W_{10}\right)$ is MDLZ transition rate from state $|0\rangle$ to $|1\rangle(|1\rangle$ to $|0\rangle)$. We emphasize that in the derivation of Eq. (20), all the expansion terms in Eq. (14), not only the notable terms, have the contribution. Equation (20) gives the evolution of the diagonal part of the system reduced density matrix under the "Markovian" approximation (see Appendix). It should be noted that we take the relaxation phenomenonally and hence it does not emerge in Hamiltonian and rate equations, while in the calculation of population we will add it. Furthermore, MDLZ transition rates obtained are the combination of Lorentzian line shapes and time dependent,

$$
\begin{gathered}
W_{01}(t)=\frac{\Delta^{2}}{2} \sum_{n, m} J_{n+m}\left(\frac{A}{\omega}\right) J_{m}\left(\frac{A}{\omega}\right) \frac{\Gamma_{2} \cos n(\omega t+\pi / 2)}{\left(\varepsilon_{0}-m \omega\right)^{2}+\Gamma_{2}^{2}}, \\
W_{10}(t)=\frac{\Delta^{2}}{2} \sum_{n, m}(-1)^{n} J_{n+m}\left(\frac{A}{\omega}\right) J_{m}\left(\frac{A}{\omega}\right) \frac{\Gamma_{2} \cos n(\omega t+\pi / 2)}{\left(\varepsilon_{0}-m \omega\right)^{2}+\Gamma_{2}^{2}},
\end{gathered}
$$

where we use the relation $J_{m}(x)=(-1)^{m} J_{-m}(x)$. Different from Eq. (9), MDLZ transition rates in Eq. (21) are unsymmetrical. Comparing Eqs. (21) and (9), we find that their difference is the additional time-dependent oscillating terms $f(t)$, which comes from the nonresonant terms. The nonresonant terms also correspond to the weak oscillation in Fig. 2(b), due to the coherence evolution. It is clear that the coherence in the nonresonant terms results into unsymmetrical MDLZ transitions, i.e., $W_{01}(t) \neq W_{10}(t)$. In addition, although the coherence is preserved in the nonresonant terms, nonresonant terms with different oscillation frequencies counteract the resonant terms which would become less obvious. Therefore, not only the decoherence but also the low-frequency driving would eliminate the multiphoton resonant fringes.

Then we have the population in state $|0\rangle$

$$
\begin{aligned}
\rho_{00}=\exp \left[\int_{0}^{t} d t^{\prime}\left(-W_{01}\left(t^{\prime}\right)-W_{10}\left(t^{\prime}\right)-\Gamma_{01}-\Gamma_{10}\right)\right] \times \\
\times\left[\int_{0}^{t} d t^{\prime}\left(W_{10}\left(t^{\prime}\right)+\Gamma_{10}\right] \times\right.
\end{aligned}
$$$$
\times \exp \left[\int_{0}^{t^{\prime}} d t^{\prime \prime}\left(-W_{01}\left(t^{\prime \prime}\right)-W_{10}\left(t^{\prime \prime}\right)-\Gamma_{01}-\Gamma_{10}\right)+1\right] .
$$

Similarly, by following the above procedure, we can extend perturbation approach to higher levels and different noise sources, which were previously explored under high- frequency driving [26,27]. Especially, MDLZ transition between higher levels can be used to analyze microwave cooling with the maximal efficiency [22].

Under the large amplitude driving, the coherence of LZS interference comes from two aspects. The first one is traditionally from the prepared initial states. The off diagonal elements of the density matrix describe this part of coherence, which is usually needed to be preserved for the application of quantum computation. The second one is the coherence induced by the crossover which acts as a beam splitter $[9,16]$. The microwave drives the state through the crossover, splitting the state into the superposition of states $|0\rangle$ and $|1\rangle$, and the system gains the coherence again. For a stationary case, the first kind has disappeared fully while the second one is created incessantly. However, it should also be noted that usually the coherence induced by the crossover only exists between two consecutive passing through the crossover within one period. There is no coherence for the processes in arbitrary periods.

To make this method reasonable, as discussed in Appendix, the coherent evolution produced by the crossover cannot produce remarkable population change in the stationary case, which means $\Delta J_{n}(\mathrm{~A} / \omega)$ has little effect in one driving period. If the tunnel coupling strength $\Delta$ is large and the decoherence is weak, the coherence will persist for a long time, producing remarkable coherent population change. Therefore, the validity of this method depends on two factors. The first one is the high frequency, i.e., $\omega \gg \Delta J_{n}(A / \omega)$, which makes coherent dynamics not have enough time to cause population change. The second one is the strong decoherence, which makes the coherence disappear quickly and produce little effect on the population, i.e., $\Gamma_{2}>\Delta J_{n}(A / \omega)$. If one of factors is satisfied, the method would be available.

For the high frequency, i.e., $\omega>\Delta \gg \Delta J_{n}(A / \omega)$, the time-dependent terms in Eq. (21) oscillate fast and can be neglected just like that in RWA. Therefore MDLZ transition Eq. (21) returns to symmetrical perturbation-RWA result Eq. (9) and is constant. In addition, as shown in the derivation, Eq. (9) can be further extended to the incoherent region $\omega<\Gamma_{2}$ and the high coherence $\Gamma_{2}<\Delta J_{n}(A / \omega)$. Furthermore, in the condition, i.e., $\omega \gg \Gamma_{2}, \Delta J_{n}(A / \omega)$ the stationary population Eq. (11) in perturbation-RWA is equivalent to Eq. (8) in RWA. To validate the perturbation-RWA result in the two regions, we have numerically solved the Bloch equations to compare with the analytical results obtained by Eq. (9) on the steady occupation probability in state $|0\rangle$, as illustrated in Fig. 3. Here we consider the frequency $\omega / 2 \pi=90 \mathrm{MHz}$ with different decoherence rates. The analytical results agree well with the numerical simulations.

For the low frequency, i.e., $\omega<\Delta$, the nonresonant terms will be comparable with the oscillating terms and cannot be neglected. In this case, perturbation-RWA result is not available and Eq. (21) must be needed. As discussed above, if the decoherence rate is small, i.e., $\Gamma_{2}<\Delta J_{n}(A / \omega) \sim W_{01}$, 

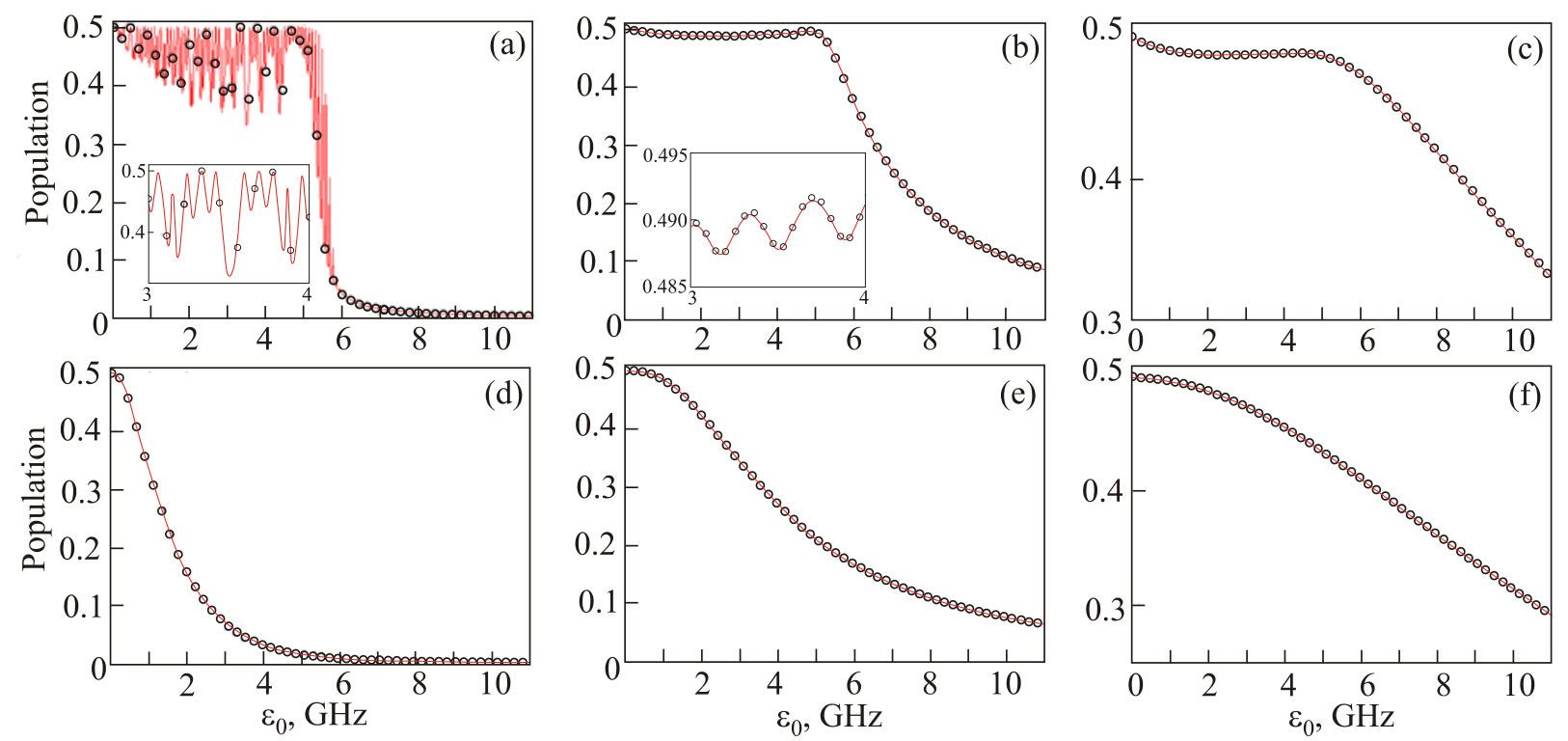

Fig. 3. (Color online) Stationary population in state $|0\rangle$ as a function of dc energy detuning $\varepsilon_{0}$ in the presence of decoherence. In all figures, $\omega / 2 \pi=90 \mathrm{MHz}, \Delta / 2 \pi=90 \mathrm{MHz}$, the interwell relaxation time $T_{1}=1 /(2 \pi \times 0.00005) \mathrm{ns}$, and the temperature $50 \mathrm{mK}$. The driving amplitude is given by $A / 2 \pi=5 \mathrm{GHz}$ (a)-(c) and $0.01 \mathrm{GHz}$ (d)-(f). The decoherence rate is given by $\Gamma_{2} / 2 \pi=3 \mathrm{MHz}$ (a) and (d), $110 \mathrm{MHz}$ (b) and (e), and $1050 \mathrm{MHz}$ (c) and (f). The red solid line shows the analytical result from Eq. (11). The open circles give the numerical results. The inset shows clearly the accordance between analytical and numerical results.

the strong coherent population evolution may break perturbation approach. Therefore in this region, for smaller frequency, perturbation approach can be used in stronger decoherence. When the decoherence is enough strong, i.e., $\Gamma_{2}>\Delta J_{n}(A / \omega)$, the coherence generated by the crossover would have no effects.

When the state is driven with the low frequency through the crossover $\Delta$, the small sweeping velocity makes the effect of nonresonant terms released. Nonreso- nant terms generate the periodic change of MDLZ transition rate with the same period of the driving, which makes the interwell relaxation participate in the dynamics and cause the change of the qubit population, i.e., $\dot{\rho}_{00}=\dot{\rho}_{11} \neq 0$, in the stationary case. Then the population would also oscillate with the frequency $\omega$. As shown in Fig. 4 , in the steady case with large amplitude $A>\varepsilon_{0}$, for the driving frequencies 10 and $1 \mathrm{MHz}$, the population in state $|0\rangle$ exhibits a periodic change [Fig. 4(a) and (b)]

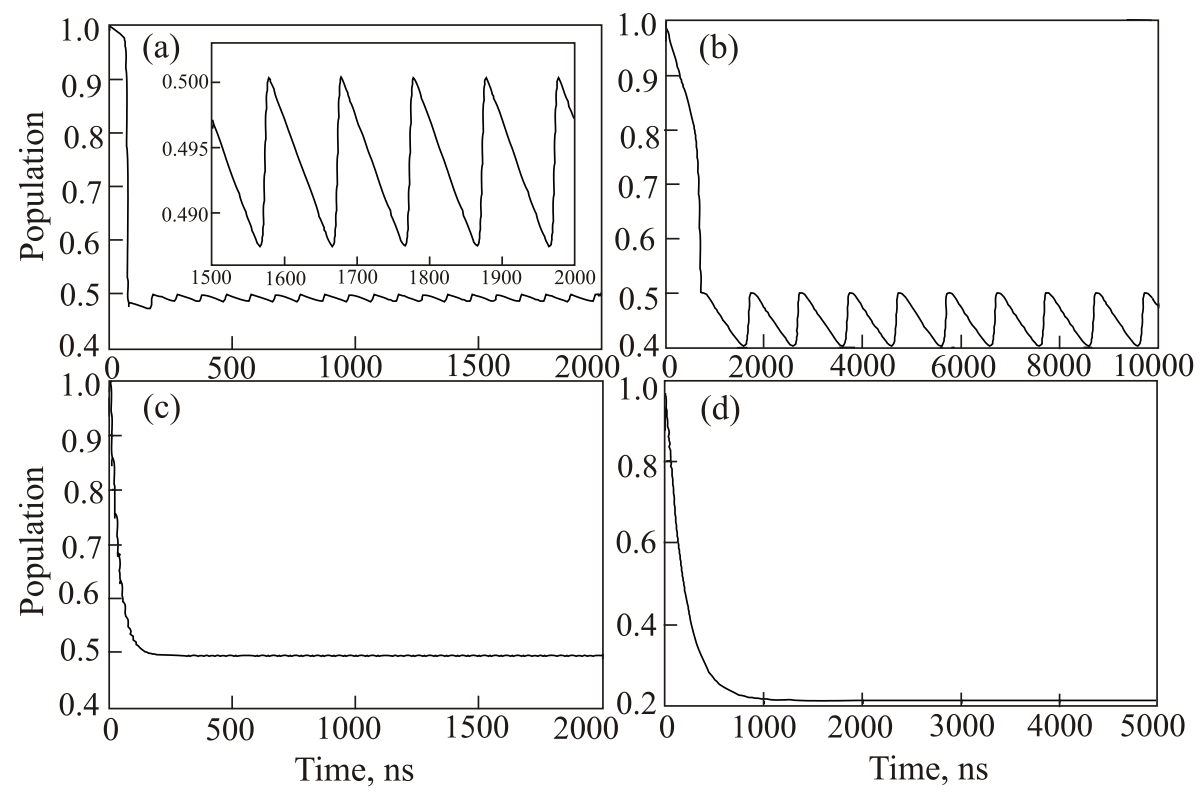

Fig. 4. Population in state $|0\rangle$ as a function of time in the presence of decoherence, assuming that the system is initially in state $|0\rangle$. In all figures, $\varepsilon_{0} / 2 \pi=4950 \mathrm{MHz}$. The amplitude is given by $A / 2 \pi=5 \mathrm{GHz}$ (a)-(c), $0.01 \mathrm{GHz}$ (d). The microwave frequency is given by $\omega / 2 \pi=10 \mathrm{MHz}(\mathrm{a}), 90 \mathrm{MHz}$ (c), $1 \mathrm{MHz}$ (b) and (d). The decoherence rate is $\Gamma_{2} / 2 \pi=110 \mathrm{MHz}$. Other parameters are identical with those in Fig. 3. The inset shows clearly the steady periodic change of population. 
while for the frequency $90 \mathrm{MHz}$ the population is always constant [Fig. 4(c)]. On the other hand, at the small amplitude, the curve becomes smooth again [Fig. 4(d)]. We can approximate $\Delta J_{n}(A / \omega)=(\Delta / n !)(A / 2 \omega)^{n}$. Small amplitude makes $\Delta J_{n}(A / \omega)$ much smaller than $\omega$ such that the high-frequency case Eq. (9) can be used. This case can also be understood simply: when the amplitude is small as illustrated, the state does not pass through the crossover and LZ transition would not occur, resulting little change of population in the steady case.

\section{Average perturbation approximation}

As discussed in Refs. 21 and 22, the low frequency $\omega$, i.e. $\Delta J_{n}(A / \omega) \lesssim \omega<\Delta$, can produce the optimal microwave cooling which is used to realize the active cooling of qubit and other artificial devises through MDLZ transitions. Although the low frequency makes the qubit population time-dependent in the stationary case, we can consider the average population over time. For the high frequency, the average population is just the steady population described by Eq. (11) while for the low frequency, the average population can be obtained by perturbation approach. However, it is complicate and not convenient to calculate the average population with Eq. (21). Therefore, we will employ average perturbation approximation (APA) based on perturbation approach in this section.

Then, we go back to the expression of $f(t)$, Eq. (19). In the stationary case, considering the time in one period, i.e., $k T<t<k T+T$, for the term $M(n, m, t)$ in $f(t)$, we have

$M(n, m, t)=J_{n+m}\left(\frac{A}{\omega}\right) J_{m}\left(\frac{A}{\omega}\right) \frac{\Gamma_{2} \cos n(\omega t+\pi / 2)}{\left(\varepsilon_{0}-m \omega\right)^{2}+\Gamma_{2}^{2}}, \quad n \neq 0$

where $M\left(n, m, k T+t^{\prime}\right)=M\left(n, m, k T+T / 2 n+t^{\prime}\right)$ with an even number $n$ and $M\left(n, m, k T+t^{\prime}\right)=M\left(n, m, k T+T-t^{\prime}\right)$ with an odd number $n$. Therefore, in one period of time, the above relation leads to

$$
\lim _{k T \rightarrow \infty} \int_{k T}^{k T+T} d t^{\prime} f\left(t^{\prime}\right)=0
$$

and we obtain the average MDLZ transition rates by averaging Eq. (21) over one period in the stationary case

$$
\begin{gathered}
\left\langle W_{10}\right\rangle=\left\langle W_{01}\right\rangle \approx \frac{1}{T} \lim _{k T \rightarrow \infty} \int_{k T}^{k T+T} d t^{\prime} W_{01}\left(t^{\prime}\right)= \\
=\frac{\Delta^{2}}{2} \sum_{n} \frac{\Gamma_{2} J_{n}^{2}(A / \omega)}{\left(\varepsilon_{0}-n \omega\right)^{2}+\Gamma_{2}^{2}} .
\end{gathered}
$$

It should be mentioned that although Eq. (24) coincides with the transition rate Eq. (9) in perturbation-RWA, the latter is the stationary transition rate under the high- frequency driving while the other is the average transition rate through averaging over the driving period under the low-frequency driving. Having obtained the transition rate, we can describe the population of system dynamics using the rate equations, where the average population $\left\langle\rho_{i i}\right\rangle$ obey

$$
\begin{gathered}
\left\langle\dot{\rho}_{00}\right\rangle=-\left(\left\langle W_{01}\right\rangle+\Gamma_{01}\right)\left\langle\rho_{00}\right\rangle+\left(\left\langle W_{10}\right\rangle+\Gamma_{10}\right)\left\langle\rho_{11}\right\rangle, \\
1=\left\langle\rho_{00}\right\rangle+\left\langle\rho_{11}\right\rangle .
\end{gathered}
$$

After the long-time system evolution, we have $\left\langle\dot{\rho}_{00}\right\rangle=\left\langle\dot{\rho}_{11}\right\rangle=0$, and the average qubit population in state $|0\rangle$ can be easily solved from Eq. (25):

$$
\left\langle\rho_{00}\right\rangle=\left(\left\langle W_{01}\right\rangle+\Gamma_{10}\right) /\left(2\left\langle W_{01}\right\rangle+\Gamma_{10}+\Gamma_{01}\right) .
$$

The rate equations used here are different from Eq. (20). Under the low-frequency driving, Eq. (20) describes the real-time dynamics with the periodic change of population. However, Eq. (26) neglects the population change within one period as shown in Figs. 4(a) and (b) and focuses on the scale of one period, in which the average population would be constant. If APA is valid, the change of qubit population must be slow on the scale of the driving period, i.e. $\omega\rangle\left\langle W_{01}\right\rangle$. If $\omega$ is larger than $\left\langle W_{01}\right\rangle$, the population change in one period is so small that

$$
\int_{t^{\prime}}^{t^{\prime}+T} W_{01} \rho_{00} d t / T \approx\left\langle\rho_{00}\right\rangle \int_{t^{\prime}}^{t^{\prime}+T} W_{01} d t / T=\left\langle\rho_{00}\right\rangle\left\langle W_{01}\right\rangle
$$

and the rate equations can be changed to the form of Eq. (25).

In order to show the region of validity for APA, we compare the numerical solutions with analytical results. The average transition rate $\left\langle W_{01}\right\rangle$ is probably less than $5 \cdot 2 \pi \mathrm{MHz}$ and generally the same order with $\Delta J_{n}(A / \omega)$. For several decoherence rates, we select the frequency $1 \mathrm{MHz}$ and obtain the average steady population, shown in Fig. 5. The deviation between the numerical and analytical results cannot be neglected and APA is not proper. Figure 4(b) shows the real-time population in state $|0\rangle$ with the numerical stimulation under the frequency $1 \mathrm{MHz}$. Comparing with the result of frequency $10 \mathrm{MHz}$ in Fig. 4(a), due to $\omega<\left\langle W_{01}\right\rangle$ the population change in Fig. 4(b) is large, demonstrating the invalidity of APA (Fig. 5).

Moreover, as shown in Figs. 5(a)-(c), at the frequency $1 \mathrm{MHz}$, when the amplitude is less than the dc detuning, which means the state does not reach the crossover, APA exhibits the good agreement with the numerical results. This is more clearly seen in Figs. 5(d)-(f) with a small amplitude $A / 2 \pi=10 \mathrm{MHz}$. Since the population change in the steady case is little [Fig. 4(d)], APA is still available. In Fig. 6, for the frequency $10 \mathrm{MHz}$, the analytical results show very good agreement for several decoherence rates, changing from the rate less than $\Delta J_{n}(A / \omega)$ to the one much larger than $\Delta$. When the amplitude is larger than the dc detuning, the population is near 0.5 , which also pro- 

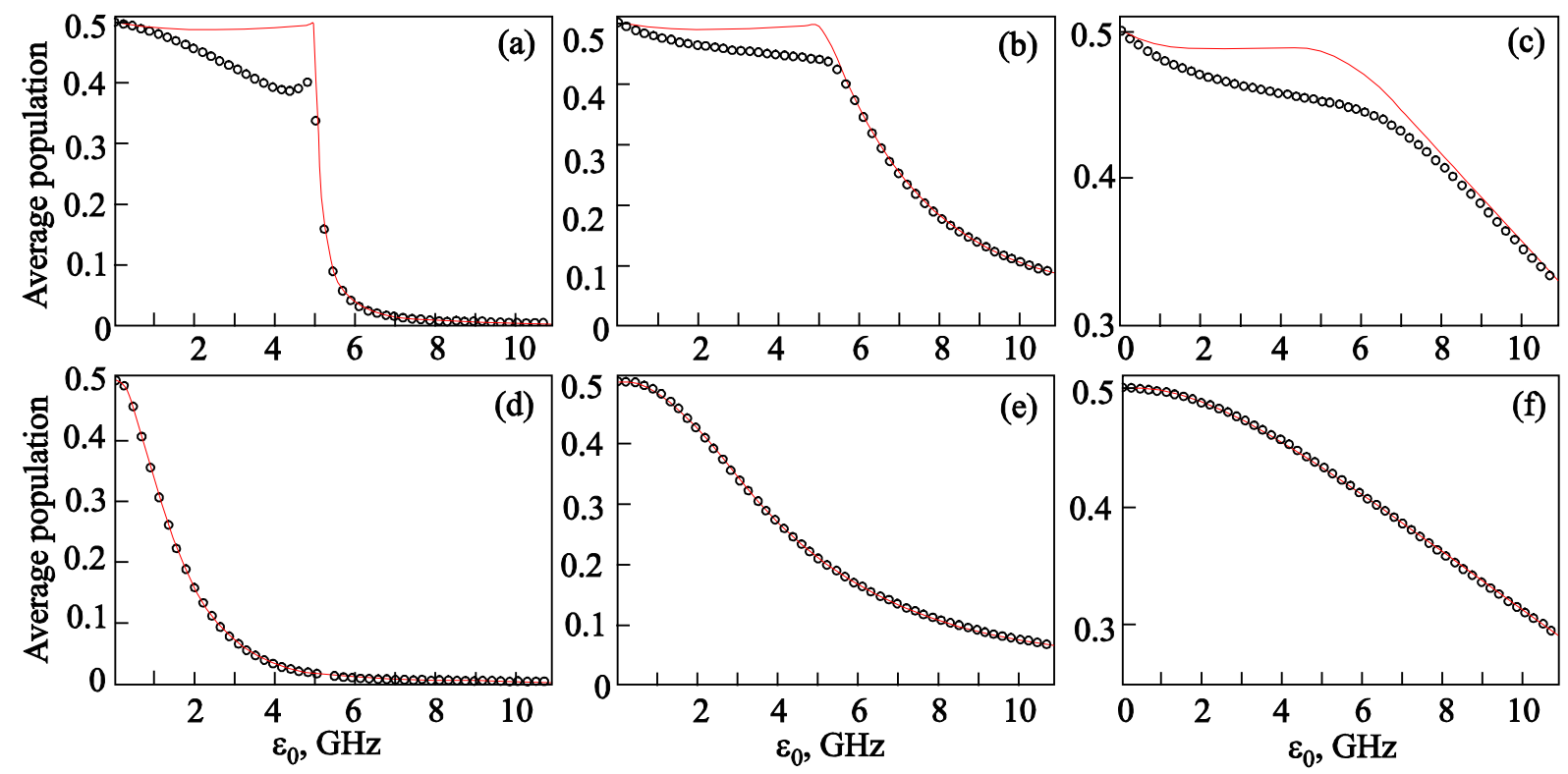

Fig. 5. (Color online) Average stationary population in state $|0\rangle$ as a function of dc energy detuning $\varepsilon_{0}$ in the presence of decoherence. In all figures, $\omega / 2 \pi=1 \mathrm{MHz}$, and $\varepsilon_{0} / 2 \pi=4950 \mathrm{MHz}$. The driving amplitude is given by $A / 2 \pi=5 \mathrm{GHz}$ (a)-(c), $0.01 \mathrm{GHz}$ (d)-(f). The decoherence rate is given by $\Gamma_{2} / 2 \pi=40 \mathrm{MHz}$ (a) and (d), $110 \mathrm{MHz}$ (b) and (e), and $1050 \mathrm{MHz}$ (c) and (f). Other parameters are identical with those in Fig. 3. The red solid line is the analytical result from Eq. (26). The open circles give the numerical results.

vides a way to determine the location of crossover with the low frequency [Figs. 6(a)-(d)]. It should be noted that with the decoherence $\Gamma_{2} / 2 \pi=3 \mathrm{MHz}$ and the frequency 10 $\mathrm{MHz}$, the agreement is not very good at the detuning $\varepsilon_{0} \approx A$. The high coherence makes the average transition rate large. As discussed in Ref. 22 , at the detuning $\varepsilon_{0} \approx A$, the transition rate reaches maximum. Therefore the frequency would be less than the average transition rate, i.e., $\omega<\left\langle W_{01}\right\rangle$, and results into the disagreement.

\section{Noncoherence approximation}

For the extremely low frequency, i.e., $\omega<$ $<\Delta J_{n}(A / \omega) \sim\left\langle W_{01}\right\rangle$, APA becomes insufficient just as shown in Fig. 5. Therefore in order to analyze this region conveniently, here we develop an analytical method based on noncoherence approximation (NCA), which will give an intuitive and clear physical picture.

When the driving frequency is high and coherent, LZS interference and multiphoton effect are clear and Bessel
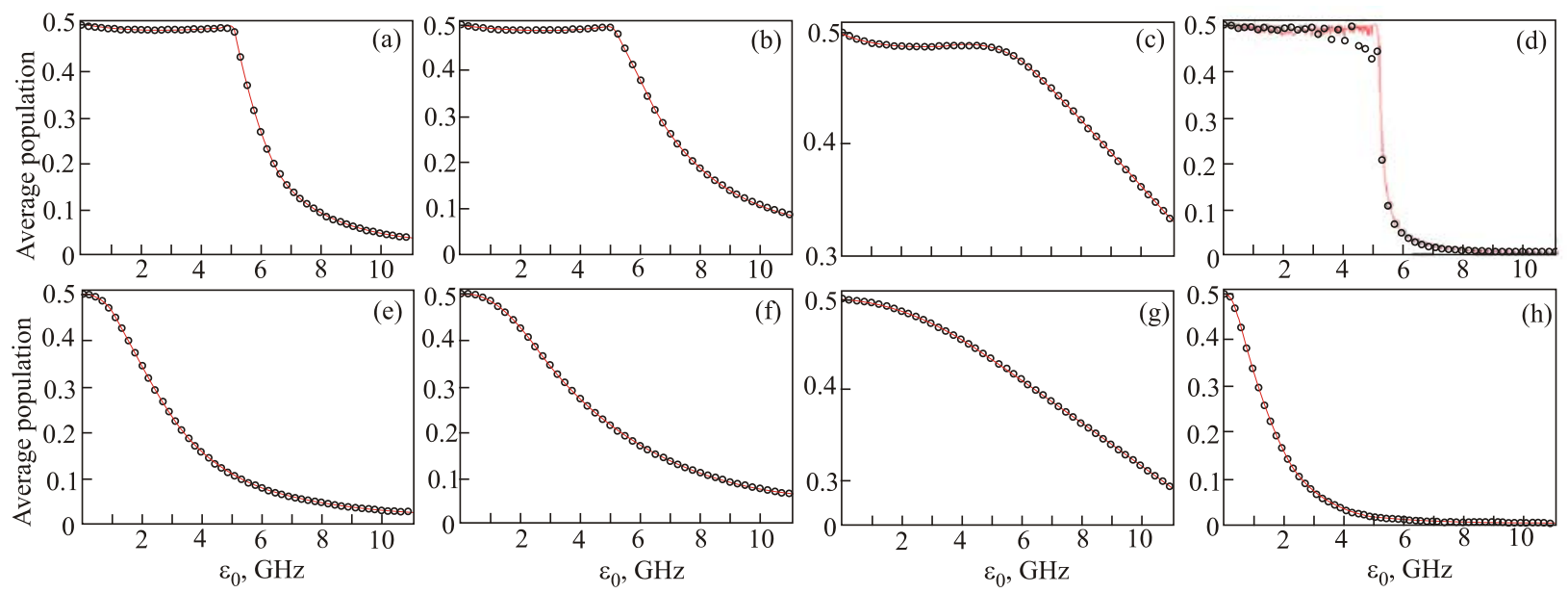

Fig. 6. (Color online) Average stationary population in state $|0\rangle$ as a function of dc energy detuning $\varepsilon_{0}$ in the presence of decoherence. In all figures, $\omega / 2 \pi=10 \mathrm{MHz}$ and $\varepsilon_{0} / 2 \pi=4950 \mathrm{MHz}$. The driving amplitude is given by $A / 2 \pi=5 \mathrm{GHz}(\mathrm{a})-(\mathrm{d}), 0.01 \mathrm{GHz}(\mathrm{e})-(\mathrm{h})$. The decoherence rate is given by $\Gamma_{2} / 2 \pi=40 \mathrm{MHz}$ (a) and (e), $110 \mathrm{MHz}$ (b) and (f), $1050 \mathrm{MHz}$ (c) and (g), $3 \mathrm{MHz}$ (d) and (h). Other parameters are identical with those in Fig. 3. The red solid line is the analytical result from Eq. (26). The open circles give the numerical results. 
functions can give a convenient picture. However, the decrease of frequency makes LZS interference gradually disappear. Therefore the description with Bessel functions would not be proper for the extremely low frequency and we return back to the sinusoidal description.

We go back to Eq. (16) and have

$$
\dot{y}(t)=\frac{\Delta^{2}}{4} \frac{d}{d t} \int_{0}^{t} d \tau^{\prime} \int_{-\tilde{t}}^{\tilde{t}} d \tau \exp \left\{i \int_{\tau^{\prime}-\tau / 2}^{\tau^{\prime}+\tau / 2} d t^{\prime}\left(\varepsilon_{0}+A \sin \omega t^{\prime}\right)-\Gamma_{2}|\tau|\right\}=
$$

$$
\begin{aligned}
& =\frac{\Delta^{2}}{4} \int_{0}^{t} d \tau \exp \left\{i \int_{t}^{t+\tau} d t^{\prime}\left(\varepsilon_{0}+A \sin \omega t^{\prime}\right)-\Gamma_{2}|\tau|\right\}+ \\
& +\frac{\Delta^{2}}{4} \int_{-t}^{0} d \tau \exp \left\{i \int_{t-\tau}^{t} d t^{\prime}\left(\varepsilon_{0}+A \sin \omega t^{\prime}\right)-\Gamma_{2}|\tau|\right\}
\end{aligned}
$$

Because of the strong decoherence, the extreme low frequency usually is much less than the decoherence rate. The decoherence makes the qubit lose coherence quickly, even though the state is split by the crossover and obtains the coherence again in each period. Hence we can approximately replace $\sin \omega t^{\prime}$ to $\sin \omega t$. Then we follow the procedure in Sec. 3, and have MDLZ transition rates

$$
W_{10}(t)=W_{01}(t)=\frac{\Delta^{2}}{2} \frac{\Gamma_{2}}{\left(\varepsilon_{0}+A \sin \omega t\right)^{2}+\Gamma_{2}^{2}} .
$$

Equation (28) is actually the result of Eq. (21) in NCA limit. Interestingly, MDLZ transition rates become symme- trical. The expression can also be understood as macroscopic resonant tunneling (MRT) $[44,46,48]$ model under the sinusoidal driving with the extremely low frequency. When the state is driven slowly to pass through each detuning, we divide the evolution into discrete time steps within which the coherence lost totally at the corresponding detunings [49]. Furthermore there is no coherence to connect time steps and the phase accumulation [9] has not existed. Since each time step is much larger than the decoherence time, we can approximately consider that MRT occurs at the detuning with the tunneling transition rate Eq. (28). Hence, the dynamics is changed from LZS interference to the periodically driven MRT and we have the rate equations

$$
\begin{gathered}
\dot{\rho}_{00}=-\left[W_{01}(t)+\Gamma_{01}\right] \rho_{00}+\left[W_{10}(t)+\Gamma_{10}\right] \rho_{11}, \\
1=\rho_{00}+\rho_{11},
\end{gathered}
$$

where $W_{01}$ is defined by Eq. (28) and $\Gamma_{01}\left(\Gamma_{10}\right)$ has the same definition with that in Eq. (7). In the evolution Eqs. (29), the periodic change makes the previous treatment $\dot{\rho}_{00}=\dot{\rho}_{11}=0$ incorrect. When the initial state is $|0\rangle$, we have the population in state $|0\rangle$

$$
\begin{gathered}
\rho_{00}=\underset{0}{\exp }\left[\int_{0}^{t} d t^{\prime}\left(-2 W_{01}\left(t^{\prime}\right)-\Gamma_{01}-\Gamma_{10}\right)\right]\left\{\int_{0}^{t} d t^{\prime}\left[W_{10}\left(t^{\prime}\right)+\Gamma_{10}\right] \times\right. \\
\quad \times \underset{0}{\left.\exp \left[\int_{0}^{t^{\prime}} d t^{\prime \prime}\left(-2 W_{01}\left(t^{\prime \prime}\right)-\Gamma_{01}-\Gamma_{10}\right)\right]+1\right\}}
\end{gathered}
$$
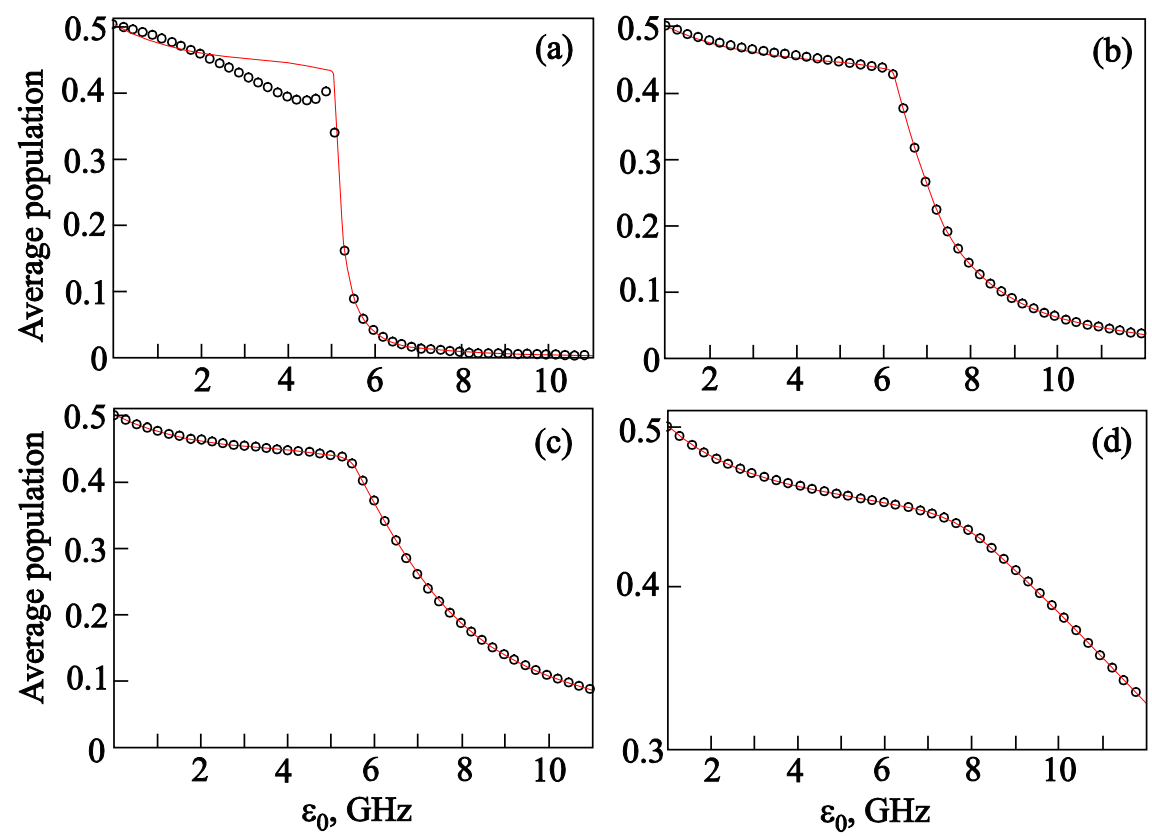

Fig. 7. (Color online) Average stationary population in state $|0\rangle$ as a function of dc energy detuning $\varepsilon_{0}$ in the presence of decoherence. In all figures, $\omega / 2 \pi=1 \mathrm{MHz}$ and $A / 2 \pi=5 \mathrm{GHz}$. The decoherence rate is $\Gamma_{2} / 2 \pi=3 \mathrm{MHz}$ (a), $40 \mathrm{MHz}$ (b), $110 \mathrm{MHz}$ (c), and $1050 \mathrm{MHz}$ (d). Other parameters are identical with those in Fig. 3. The red solid line shows the analytical result in Eq. (30) through noncoherence approximation. The open circles give the numerical results. 
The limit of perturbation approach also has function on NCA, which requires the strong decoherence, $\Gamma_{2}>$ $>\Delta J_{n}(A / \omega) \sim\left\langle W_{01}\right\rangle$, where $\left\langle W_{01}\right\rangle$ is defined by Eq. (24). Moreover NCA needs the extremely low frequency compared with the strong decoherence. Therefore, in this region, this method is reasonable with the condition $\omega<\left\langle W_{01}\right\rangle<\Gamma_{2}$. In the frequencies where the average perturbation theory fails, we use Eq. (30) to reconsider the average population in the steady situation as shown in Fig. 7. At the decoherence rate $\Gamma_{2} / 2 \pi=3 \mathrm{MHz}$, the agreement is not good, because the decoherence rate is comparable with the driving frequency. However, for other decoherence rates, the numerical stimulations agree well with the analytical results. Furthermore, we consider the real-time evolution and compare it with the numerical stimulation (Fig. 8). As the frequency is comparable or larger than the decoherence rate, NCA will be insufficient [Fig. 8(b)].

From perturbation-RWA to NCA, we can find the transition from quantum to classic effect of the microwave with the frequency decreasing. For the high-frequency driving, being in a coherent state and with high photon numbers, the microwave behaves as the quantized electromagnetic field exchanging photons with the qubit. At the same time, the large microwave amplitude forms LZS interferometry through the crossover, modulating the intensi-
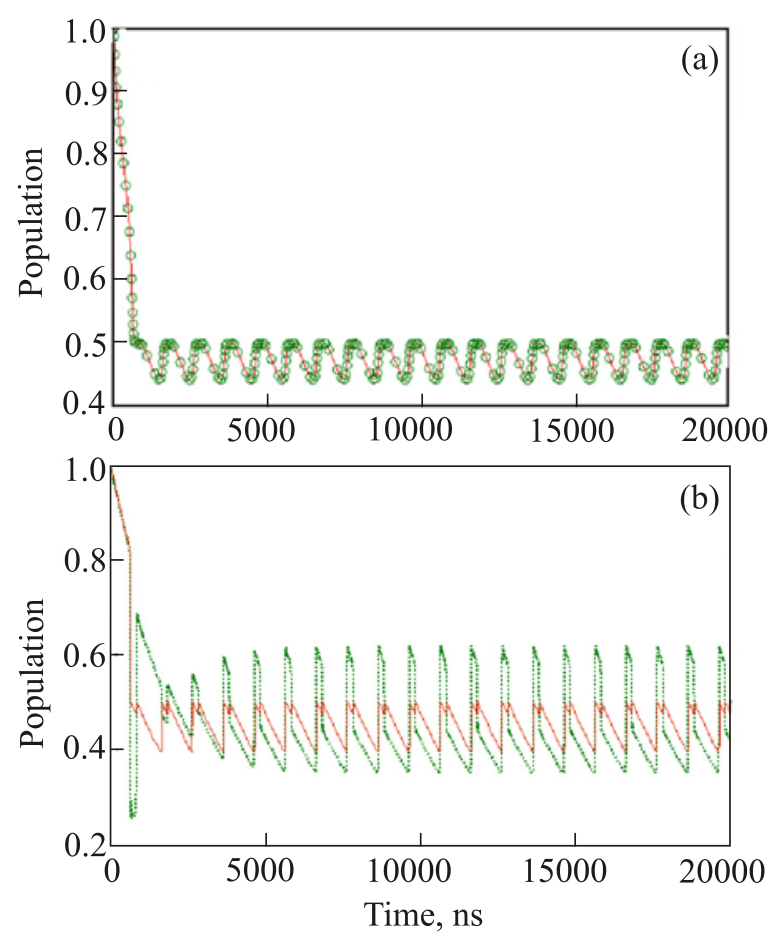

Fig. 8. (Color online) Population in state $|0\rangle$ as a function of time in the presence of decoherence, assuming that the system is initially in the state $|0\rangle$. In both figures, $\omega / 2 \pi=1 \mathrm{MHz}$, $\varepsilon_{0} / 2 \pi=4125 \mathrm{MHz}$, and $A / 2 \pi=5 \mathrm{GHz}$. The decoherence rate is given by $\Gamma_{2} / 2 \pi=1050 \mathrm{MHz}$ (a) and $3 \mathrm{MHz}$ (b). Other parameters are identical with those in Fig. 3 . The red line shows the results from the noncoherence approximation. The open circles and dotted line give the numerical results. ty of the $n$-photon resonance. In this case MDLZ transition Eq. (9) is based on perturbation-RWA and the nonresonant terms in the transition rate can be neglected. With the frequency further decreasing, the decoherence becomes stronger relative to the driving frequency and the nonresonant terms are comparable with the resonant ones, which makes the photons resonances as well as the modulation from LZS interferometry vanish gradually. Then we have to use Eq. (21) to describe MDLZ transition. For the low frequency, in the scale of one driving period, the resonant information can still be notable and we can use APA to obtain the average population. For the extremely low frequency compared with the decoherence, the microwave can finally be treated as a classic field and the dynamic process is changed to the periodically driven MRT as shown in Eq. (30).

To sum up, we plot the average stationary population obtained from analytical calculation and numerical stimulation as shown in Fig. 9(a). The different approximations of perturbation approach cover most of parameter space. Figure 9(b) is the detail of Fig. 9(a) at the decoherence rate $\Gamma_{2} / 2 \pi=110 \mathrm{MHz}$. We show the applicable regions for these approximations in the average stationary population in Fig. 9(c). The circles mark where perturbation-RWA holds. In the red shaded region I, a high-frequency coherent field is used to realize multiphoton resonance $[9,19]$. With the frequency further decreasing, we enter $\left\langle W_{01}\right\rangle \lesssim \omega<\Delta$. In the stationary state the qubit population exhibits the periodic oscillation which cannot be explained using perturbation-RWA. Now APA is applicable, marked with blue squares region III in Fig. 9(c). In the green shaded region II, the low-frequency driving is able to generate microwave cooling and the optimal microwave cooling was realized by Valenzuela et al. [21]. Then for the extremely low frequency $\omega<\left\langle W_{01}\right\rangle<\Gamma_{2}$, APA is also unsuccessful. NCA can describe the behavior, as marked by the black ellipses. In the blue shaded region III, which is not covered by any before mentioned approximation, the high coherence and low frequency make it possible and convenient to realize adiabatic LZ process or LZS interference by a single crossing $[16,50,51]$ with large amplitude. It should be emphasized that the region in Fig. 9(c) would change with the amplitude just as shown in Fig. 5. For the amplitude larger than the dc detuning, the small frequency locates in NCA region while for the amplitude less than the dc detuning, this frequency may move to APA region.

\section{Multilevel system}

Our approach gives a simple but effective physical picture, which enables us easily to extend the discussion to a multilevel flux qubit [Fig. 10(a)] [15,27]. In this section, based on the approximations we will discuss the lower limit of driving frequency in effective microwave driven cooling. 


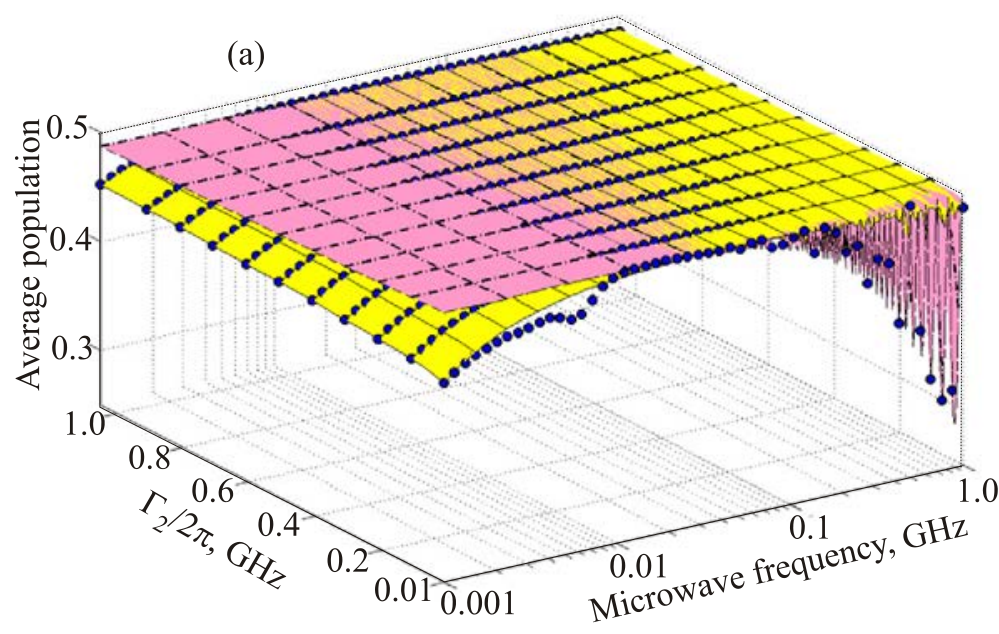

(b)

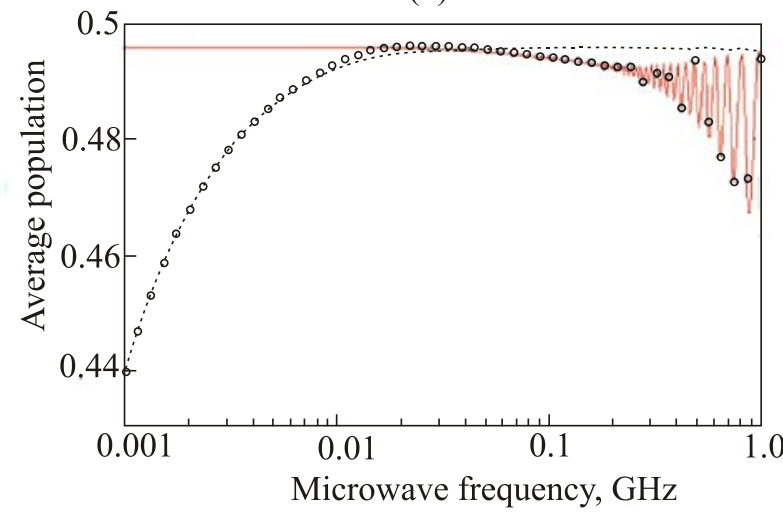

(c)

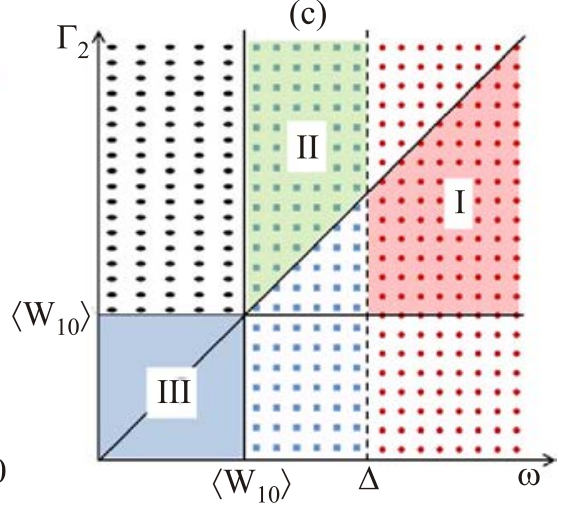

Fig. 9. (Color online) (a) Population in state $|0\rangle$ of TLS described by Fig. 1 as functions of microwave frequency and decoherence rate. $\varepsilon_{0} / 2 \pi=4950 \mathrm{MHz}$ and $A / 2 \pi=5 \mathrm{GHz}$. Other parameters are the same with those in Fig. 3. The red surface with dashed line is obtained from the average perturbation approximation. The yellow surface with solid line is resulted from the noncoherence approximation. The blue solid circles are from the data of numerical stimulation. (b) The detail of (a) at the decoherence rate $\Gamma_{2} / 2 \pi=110 \mathrm{MHz}$. (c) Regions of validity for different approximations. The axes are the driving frequency and decoherence rate, respectively. Perturbation-RWA region is described by the condition $\omega>\Delta$ and it is marked by the red circles. The average perturbation approximation region is described by the condition $\left\langle W_{10}\right\rangle<\omega<\Delta$ and it is represented by the blue squares. The noncoherence approximation region is shown by the condition $\omega<\left\langle W_{10}\right\rangle<\Gamma_{2}$ and it is marked by the black ellipses.

In Ref. 21, it was found that a lower driving frequency realized better cooling effect and at $5 \mathrm{MHz}$ the optimal cooling appeared. The driving microwave frequency used is so low that one does not need the high-frequency microwave generator and microwave line to realize active cooling. On the other hand, generally, if the microwave frequency is too low, there would not be the cooling effect on the qubit. Therefore, there exists a minimum frequency to produce the effective microwave cooling. Considering the three-level system consisting of an "internal" qubit $(|1\rangle$ and $|0\rangle)$ and an "internal” oscillator like state $|2\rangle$, as shown in Fig. 10(a), we rewrite the rate equations (29) to three-level form

$$
\begin{gathered}
\dot{\rho}_{00}=-\left[W_{01}(t)+\Gamma_{01}+W_{02}(t)\right] \rho_{00}+ \\
+\left[W_{10}(t)+\Gamma_{10}\right] \rho_{11}+W_{20}(t) \rho_{22}, \\
\dot{\rho}_{11}=\left[W_{01}(t)+\Gamma_{01}\right] \rho_{00}-\left[W_{10}(t)+\Gamma_{10}\right] \rho_{11}+\Gamma_{21} \rho_{22}, \\
1=\rho_{00}+\rho_{11}+\rho_{22},
\end{gathered}
$$

where $\Gamma_{21}$ is the intrawell relaxation from state $|2\rangle$ to $|1\rangle$.
For the low frequency as discussed in Sec. 4, APA can be applicable and we have

$$
W_{20}(t)=W_{02}(t)=\frac{\Delta_{20}^{2}}{2} \sum_{n} \frac{\left(\Gamma_{2}+\Gamma_{21} / 2\right) J_{n}^{2}(A / \omega)}{\left(\varepsilon_{0}^{\prime}-n \omega\right)^{2}+\left(\Gamma_{2}+\Gamma_{21} / 2\right)^{2}},
$$

and

$$
W_{01}(t)=W_{10}(t)=\frac{\Delta_{01}^{2}}{2} \sum_{n} \frac{\Gamma_{2} J_{n}^{2}(A / \omega)}{\left(\varepsilon_{0}-n \omega\right)^{2}+\Gamma_{2}^{2}}
$$

where

$$
\varepsilon_{0}^{\prime}=\left(\left|m_{0}\right|+\left|m_{2}\right|\right)\left(\delta \Phi_{d c}-\Phi_{20}\right), \quad \varepsilon_{0}=\left(\left|m_{0}\right|+\left|m_{1}\right|\right) \delta \Phi_{d c},
$$

and $\Phi_{20}$ is the flux detuning where the crossover $\Delta_{20}$ is reached. It should be mentioned that Eqs. (32) and (33) actually express the average transition rate. Then for the extremely low frequency as discussed in Sec. 5, we have

$$
W_{20}(t)=W_{02}(t)=\frac{\Delta_{20}^{2}}{2} \frac{\Gamma_{2}+\Gamma_{21} / 2}{\varepsilon^{*}(t)^{2}+\left(\Gamma_{2}+\Gamma_{21} / 2\right)^{2}},
$$



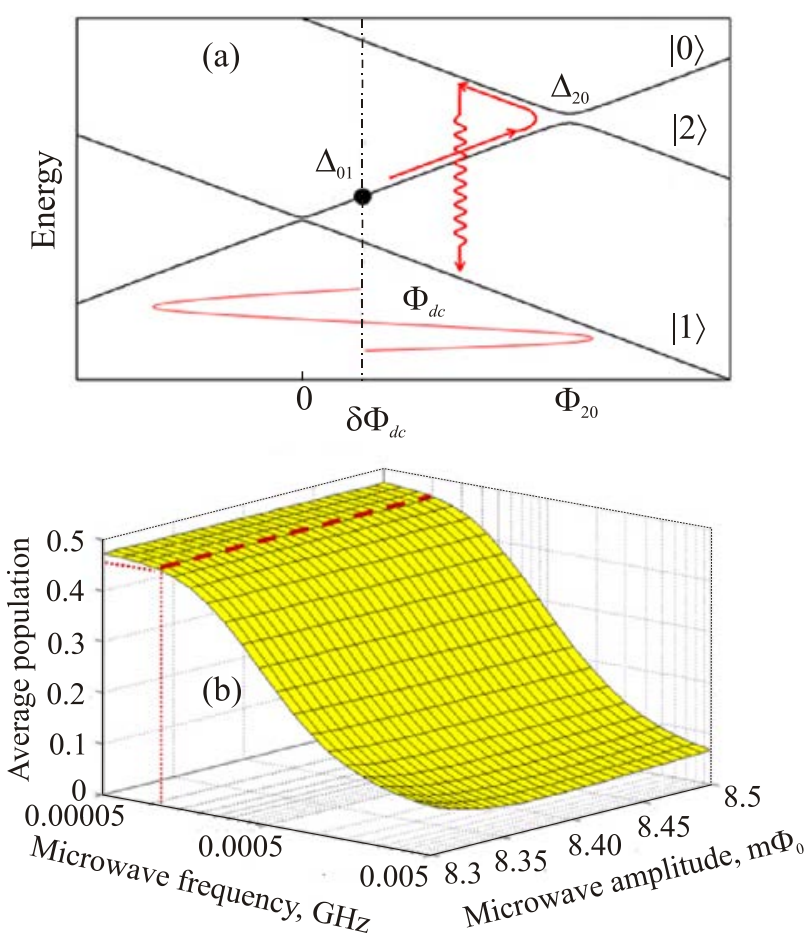

Fig. 10. (Color online) (a) Schematic energy diagram of a multilevel flux qubit. Red solid curve represents the microwave $\Phi_{a c}$. The dashed black line marks a particular static flux detuning $\delta \Phi_{d c}$. State $|0\rangle$ is in the left well; $|1\rangle$ and $|2\rangle$ are in the right well. The red solid path describes the cooling with the population in state $|0\rangle$ transferred to $|1\rangle$. The locations of the crossovers $\Delta_{01}$ and $\Delta_{20}$ are $0 \mathrm{~m} \Phi_{0}$ and $8.4 \mathrm{~m} \Phi_{0}$, respectively. The diabatic energy-level slope $\left|m_{0}\right|\left(\left|m_{1}\right|\right)=2 \pi \times 1.44 \mathrm{GHz} / \mathrm{m}_{0}$, and $\left|m_{2}\right|=2 \pi \times 1.09 \mathrm{GHz} / \mathrm{m}_{0} . \Delta_{01} / 2 \pi=0.013 \mathrm{GHz}, \Delta_{02} / 2 \pi=$ $=0.09 \mathrm{GHz}$. The parameters used above are from the experiment [15]. In calculation, $\Gamma_{2} / 2 \pi=0.06 \mathrm{GHz}, \Gamma_{21} / 2 \pi=0.1 \mathrm{GHz}$ [27], and the interwell relaxation time $T_{1}=1 /(2 \pi \times 0.00005) \mathrm{ns}(\mathrm{b})$. The yellow surface with solid line shows the population in state $|0\rangle$ versus microwave frequency and amplitude at dc flux detuning $\delta \Phi_{d c}=0.05 \mathrm{~m} \Phi_{0}$. The temperature is $50 \mathrm{mK}$. The dashed line indicates the population in equilibrium at this detuning.

and

$$
W_{01}(t)=W_{10}(t)=\frac{\Delta_{01}^{2}}{2} \frac{\Gamma_{2}}{\varepsilon(t)^{2}+\Gamma_{2}^{2}}
$$

where

$$
\varepsilon(t)=\left(\left|m_{0}\right|+\left|m_{1}\right|\right) \delta \Phi(t)
$$

and

$$
\varepsilon^{*}(t)=\left(\left|m_{0}\right|+\left|m_{2}\right|\right)\left[\delta \Phi(t)-\Phi_{20}\right] .
$$

Here, we focus the extremely low-frequency region. Since the analytical solution is too complex to extract a clear physical picture, we do not write it out explicitly here. Figure 10 (b) shows the population in state $|0\rangle$ at the dc flux detuning $\delta \Phi_{d c}=0.05 \mathrm{~m} \Phi_{0}$ as functions of frequency and amplitude. The thick dashed line marks the population at equilibrium with the temperature $50 \mathrm{mK}$. It can be seen that when the frequency is less than $0.1 \mathrm{MHz}$, microwave cooling would be ineffective.

In the extremely low frequency, the interwell relaxation changes the qubit population within one period as shown in Eq. (31). When the state is driven through the crossover $\Delta_{20}$, LZ transition would transfer the population from state $|0\rangle$ to $|2\rangle$, which then quickly relaxes into state $|1\rangle$. As the states is driven back to the initial detuning, the interwell relaxation warms the qubit again if the driving frequency is less than the repopulation rate of the warm process, i.e., $\Gamma_{10}+\Gamma_{01}$, which is approximately $2 \Gamma_{10}$ at the small dc detuning. The analysis agrees with the result in Fig. 10(b).

\section{Conclusion}

In this article we have studied the population evolution of a strongly driven superconducting qubit of the long-time dynamics. First of all, we use perturbation approach, which considers high order perturbation expansion and nonresonant terms, to obtain the time dependent unsymmetrical MDLZ transitions. Moreover, we point out that this approach has a high validity in the case where the influence of coherence resulted from $\Delta$ is small with the high frequency or strong decoherence. Then for different frequencies, we apply several approximations to discuss the system dynamics.

For the high frequency, i.e., $\omega>\Delta$, MDLZ transition rate in perturbation approach is constant and leads to perturbation-RWA result. In previous works $[9,19]$ perturbation-RWA has been proven successful in the coherent region under the strong decoherence, i.e., $\omega>\Delta, \Gamma_{2}$ and $\Gamma_{2} \gg W_{01}$. Besides this region, we show perturbationRWA result can also be used in the incoherent case where the frequency is smaller than the decoherence rate, $\Gamma_{2}>\omega$, and even the high-coherence region $\Gamma_{2}<W_{01}$.

With the frequency further decreasing to the region of the optimal microwave cooling, $\left\langle W_{10}\right\rangle \lesssim \omega<\Delta$, since the coherence has effect on the nonresonant terms and nonresonant terms are comparable with the resonant terms, the qubit population exhibits periodic oscillation which cannot be addressed by perturbation-RWA. In this case, we study the average stationary population in the low-frequency driving. Then we employ APA by averaging MDLZ transition rate of perturbation approach with time. Although the analytical expression is the same with that of perturbationRWA, they have different physical pictures. In perturbationRWA, nonresonant terms have no effects and can be neglected, while in APA the nonresonant terms result oscillations and can be averaged over to obtain the average population. Therefore in APA, we do not take account into the realtime dynamics, but consider one period as the unit time. 
Then in the extremely low frequency, i.e., $\omega<\left\langle W_{10}\right\rangle<\Gamma_{2}$, where APA has failed, we propose NCA by dividing the evolution into discrete time steps during which the coherence is lost totally. We show the dynamic process equivalent to the periodically driven MRT, giving a convenient and intuitive picture. In addition, in the multilevel system, based on this approximation we examine the possibility of employing the extremely low frequency driving as a useful tool to realize microwave cooling and prove that there exists a lower limit of frequency for effective microwave cooling. The different approximations of perturbation approach cover most of parameter space when describing the average population [Fig. 9(c)].

Our theory offers the effective analytical description of the driven dissipative TLS in the stationary case, including real-time and average situation. The good agreement of our theory with the numerical results shows the validity of the approximations used. By shifting these approximations, we also show the transition from quantum regime to classic regime about the interaction between qubit and microwave, with dynamics changing from LZS interference to periodically driven MRT. This could enhance the understanding to the influence of decoherence on the qubit. Furthermore, our results can be applied to understand the behavior of other systems with similar structure. Especially, our theory in the low frequency can be used to analyze the optimal microwave cooling of qubit as well as other artificial device such as nano mechanical resonators.

\section{Acknowledgment}

Thanks to S.O. Valenzuela, S.N. Shevchenko and M.H.S. Amin for useful discussions.

This work was supported in part by the State Key Program for Basic Researches of China (2011CB922104, 2011CBA00205), the NSFC (11274156, 91021003), the Natural Science Foundation of Jiangsu Province (BK2010012), and PAPD.

\section{Appendix. Derivation of Eq. (14)}

In this Appendix, we will accept the straightforward physical picture for intuitive understanding. Assuming that the system is initialized in state $|0\rangle$, first of all, we try to calculate the population in state $|0\rangle$, i.e., $\rho_{00}(t)$. In a short time interval, i.e., $t<1 /\left[\Delta J_{n}(A / \omega)\right]$, considering the perturbation expansion of $U_{V}$ in $V(\tau)$ up to the second order term, we have

$$
\begin{gathered}
\rho_{00}(t)=\operatorname{Tr}_{B}\left\{\left\langle 0\left|\rho_{S B}(t)\right| 0\right\rangle\right\}=\operatorname{Tr}_{B}\left\{\left\langle 0\left|U_{V}(t, 0) \rho_{S B}(0) U_{V}^{\dagger}(t, 0)\right| 0\right\rangle\right\}= \\
=\operatorname{Tr}_{B}\left\{\left\langle 0\left|\rho_{S B}(0)\right| 0\right\rangle\right\}-\operatorname{Tr}_{B}\left\{\int_{0}^{t} \int_{0}^{\tau} d \tau d \tau^{\prime}\left\langle 0\left|V(\tau) V\left(\tau^{\prime}\right) \rho_{S B}(0)\right| 0\right\rangle\right\}-\operatorname{Tr}_{B}\left\{\int_{0}^{t} \int_{0}^{\tau} d \tau d \tau^{\prime}\left\langle 0\left|\rho_{S B}(0) V^{\dagger}\left(\tau^{\prime}\right) V^{\dagger}(\tau)\right| 0\right\rangle\right\}
\end{gathered}
$$

We substitute $V(t)$ into Eq. (A.1) and have

$$
\begin{gathered}
\rho_{00}(t)=1-\int_{0}^{t} \int_{0}^{\tau} d \tau d \tau^{\prime} K_{1}\left\langle U_{-}^{\dagger}(\tau) U_{+}(\tau) U_{+}^{\dagger}\left(\tau^{\prime}\right) U_{-}\left(\tau^{\prime}\right)\right\rangle-\int_{0}^{t} \int_{0}^{\tau} d \tau d \tau^{\prime} K_{2}\left\langle U_{-}^{\dagger}\left(\tau^{\prime}\right) U_{+}\left(\tau^{\prime}\right) U_{+}^{\dagger}(\tau) U_{-}(\tau)\right\rangle= \\
=1-2 \int_{0}^{t} \int_{0}^{\tau} d \tau d \tau^{\prime} K_{1}\left\langle U_{-}^{\dagger}(\tau) U_{+}(\tau) U_{+}^{\dagger}\left(\tau^{\prime}\right) U_{-}\left(\tau^{\prime}\right)\right\rangle,
\end{gathered}
$$

where

$$
K_{1}=\sum_{n, m} J_{n}\left(\frac{A}{\omega}\right) J_{m}\left(\frac{A}{\omega}\right) \mathrm{e}^{i\left(\varepsilon_{0}-m \omega\right) \tau-i\left(\varepsilon_{0}-n \omega\right) \tau^{\prime}+i n \pi / 2-i m \pi / 2}
$$

and

$$
K_{2}=\sum_{n, m} J_{n}\left(\frac{A}{\omega}\right) J_{m}\left(\frac{A}{\omega}\right) \mathrm{e}^{-i\left(\varepsilon_{0}-m \omega\right) \tau+i\left(\varepsilon_{0}-n \omega\right) \tau^{\prime}-i n \pi / 2+i m \pi / 2} .
$$

In the above derivation, we also obtain

$$
\operatorname{Tr}_{B}\left\{\left\langle 0\left|\int_{0}^{t} \int_{0}^{\tau} d \tau d \tau^{\prime} V(\tau) V\left(\tau^{\prime}\right) \rho_{S B}(0)\right| 0\right\rangle\right\}=\operatorname{Tr}_{B}\left\{\left\langle 0\left|\int_{0}^{t} \int_{0}^{\tau} d \tau d \tau^{\prime} \rho_{S B}(0) V^{\dagger}\left(\tau^{\prime}\right) V^{\dagger}(\tau)\right| 0\right\rangle\right\}
$$

Moreover $2 \operatorname{Tr}_{B}\left\{\left\langle 0\left|\int_{0}^{t} \int_{0}^{\tau} d \tau d \tau^{\prime} V(\tau) V\left(\tau^{\prime}\right) \rho_{S B}(0)\right| 0\right\rangle\right\}$ just describes the reduced population in state $|0\rangle$ in the short time interval.

Then we turn to high order expansion. It should be mentioned for simpleness in the below derivation we only consider the first four terms in Eq. (13) and higher order terms can be treated with the similar process. We have 


$$
\begin{aligned}
\rho_{11}(t)=\operatorname{Tr}_{B}\left\{\left\langle 1\left|U_{V}(t, 0) \rho_{S B}(0) U_{V}^{\dagger}(t, 0)\right| 1\right\rangle\right\}=\int_{0}^{t} \int_{0}^{t} d \tau d \tau^{\prime} \operatorname{Tr}_{B}\left\{\left\langle 1\left|V(\tau) \rho_{S B}(0) V^{\dagger}\left(\tau^{\prime}\right)\right| 1\right\rangle\right\}- \\
\quad-\int_{0}^{t} \int_{0}^{t} \int_{0}^{t_{1}} \int_{0}^{t_{2}} d t_{4} d t_{1} d t_{2} d t_{3} \operatorname{Tr}_{B}\left\{\left\langle 1\left|V\left(t_{4}\right) \rho_{S B}(0) V^{\dagger}\left(t_{3}\right) V^{\dagger}\left(t_{2}\right) V^{\dagger}\left(t_{1}\right)\right| 1\right\rangle\right\}- \\
\quad-\int_{0}^{t} \int_{0}^{t_{1}} \int_{0}^{t_{2}} \int_{0}^{t} d t_{1} d t_{2} d t_{3} d t_{4} \operatorname{Tr}_{B}\left\{\left\langle 1\left|V\left(t_{1}\right) V\left(t_{2}\right) V\left(t_{3}\right) \rho_{S B}(0) V^{\dagger}\left(t_{4}\right)\right| 1\right\rangle\right\}+\ldots
\end{aligned}
$$

Considering that the last two terms in Eq. (A.3) is equivalent, we can write Eq. (A.3) as

$$
\begin{aligned}
& \rho_{11}(t)= \int_{0}^{t} \int_{0}^{t} d \tau d \tau^{\prime} \operatorname{Tr}_{B}\left\{\left\langle 1\left|V(\tau) \rho_{S B}(0) V^{\dagger}\left(\tau^{\prime}\right)\right| 1\right\rangle\right\}-2 \iint_{0}^{t} \int_{0}^{t} \int_{0}^{t_{1}} d t_{4} d t_{1} d t_{2} d t_{3} \operatorname{Tr}_{B}\left\{\left\langle 1\left|V\left(t_{4}\right) \rho_{S B}(0) V^{\dagger}\left(t_{3}\right) V^{\dagger}\left(t_{2}\right) V^{\dagger}\left(t_{1}\right)\right| 1\right\rangle\right\}= \\
&= \int_{0}^{t} \int_{0}^{t} d \tau d \tau^{\prime} \operatorname{Tr}_{B}\left\{\left\langle 1\left|V(\tau) \rho_{S B}(0) V^{\dagger}\left(\tau^{\prime}\right)\right| 1\right\rangle\right\}-2 \int_{0}^{t} \int_{0}^{t_{1}} \int_{0}^{t_{2}} \int_{0}^{t_{2}} d t_{1} d t_{2} d t_{3} d t_{4} \operatorname{Tr}_{B}\left\{\left\langle 1\left|V\left(t_{4}\right) \rho_{S B}(0) V^{\dagger}\left(t_{3}\right) V^{\dagger}\left(t_{2}\right) V^{\dagger}\left(t_{1}\right)\right| 1\right\rangle\right\}- \\
&-2 \int_{0}^{t} \int_{0}^{t} \int_{0}^{t_{1} t_{2}} \int_{0}^{t_{2}} d t_{1} d t_{4} d t_{2} d t_{3} \operatorname{Tr}_{B}\left\{\left\langle 1\left|V\left(t_{4}\right) \rho_{S B}(0) V^{\dagger}\left(t_{3}\right) V^{\dagger}\left(t_{2}\right) V^{\dagger}\left(t_{1}\right)\right| 1\right\rangle\right\}+\ldots
\end{aligned}
$$

Then we find that the system population rate change is given by

$$
\begin{gathered}
\dot{\rho}_{11}(t)=\int_{0}^{t} d \tau \operatorname{Tr}_{B}\left\{\left\langle 1\left|V(t) \rho_{S B}(0) V^{\dagger}\left(\tau^{\prime}\right)\right| 1\right\rangle\right\}+\int_{0}^{t} d \tau \operatorname{Tr}_{B}\left\{\left\langle 1\left|V(\tau) \rho_{S B}(0) V^{\dagger}(t)\right| 1\right\rangle\right\}- \\
-2 \int_{0}^{t} \int_{0}^{t_{2}} \int_{0}^{t_{2}} d t_{2} d t_{3} d t_{4} \operatorname{Tr}_{B}\left\{\left\langle 1\left|V\left(t_{4}\right) \rho_{S B}(0) V^{\dagger}\left(t_{3}\right) V^{\dagger}\left(t_{2}\right) V^{\dagger}(t)\right| 1\right\rangle\right\}-2 \int_{0}^{t} \int_{0}^{t_{2}} \int_{t_{2}}^{t} d t_{2} d t_{3} d t_{4} \operatorname{Tr}_{B}\left\{\left\langle 1\left|V\left(t_{4}\right) \rho_{S B}(0) V^{\dagger}\left(t_{3}\right) V^{\dagger}\left(t_{2}\right) V^{\dagger}(t)\right| 1\right\rangle\right\}- \\
-2 \int_{0}^{t} \int_{0}^{t} \int_{0}^{t_{2}} d t_{1} d t_{2} d t_{3} \operatorname{Tr}_{B}\left\{\left\langle 1\left|V(t) \rho_{S B}(0) V^{\dagger}\left(t_{3}\right) V^{\dagger}\left(t_{2}\right) V^{\dagger}\left(t_{1}\right)\right| 1\right\rangle\right\}+\ldots
\end{gathered}
$$

Combining the equivalent terms in Eq. (A.5), we have

$$
\begin{gathered}
\dot{\rho}_{11}(t)=\operatorname{Tr}_{B}\left\{2 \int_{0}^{t} d \tau\left\langle 1\left|V(t) \rho_{S B}(0) V^{\dagger}(\tau)\right| 1\right\rangle\right\}-\operatorname{Tr}_{B}\left\{2 \int_{0}^{t} d t_{2}\left\langle 1\left|\left[\int_{0}^{t_{2}} \int_{0}^{t_{2}} d t_{3} d t_{4} V\left(t_{4}\right) \rho_{S B}(0) V^{\dagger}\left(t_{3}\right)\right] V^{\dagger}\left(t_{2}\right) V^{\dagger}(t)\right| 1\right\rangle\right\}- \\
\quad-\operatorname{Tr}_{B}\left\{2 \int_{0}^{t} d t_{1}\left\langle 1\left|V(t)\left[2 \int_{0}^{t_{1}} \int_{0}^{t_{2}} d t_{2} d t_{3} \rho_{S B}(0) V^{\dagger}\left(t_{3}\right) V^{\dagger}\left(t_{2}\right)\right] V^{\dagger}\left(t_{1}\right)\right| 1\right\rangle\right\}+\ldots
\end{gathered}
$$

where

$$
\int_{0}^{t_{2}} \int_{0}^{t_{2}} d t_{3} d t_{4} V\left(t_{4}\right) \rho_{S B}(0) V^{\dagger}\left(t_{3}\right)
$$

describes the population transferred from state $|0\rangle$ to $|1\rangle$ in the time interval $t_{2}$. Comparing with Eqs. (A.1) and (A.2), we can find that the second term in Eq. (A.6) characterizes the population rate transferred from state $|1\rangle$ to $|0\rangle$ based on the population transferred from state $|0\rangle$. Similarly

$$
2 \int_{0}^{t_{1}} \int_{0}^{t_{2}} d t_{2} d t_{3} \rho_{S B}(0) V^{\dagger}\left(t_{3}\right) V^{\dagger}\left(t_{2}\right)
$$

describes the reduced population in state $|0\rangle$ in the time interval $t_{1}$ and the third term in Eq. (A.6) describes the reduced population rate from state $|0\rangle$ to $|1\rangle$ due to the decrease of population in state $|0\rangle$. Then we replace the integration limit $t_{2}$ in Eq. (A.6) to $t$, which is similar with the widely known Markovian approximation dealing with the population transition caused by the coupling to the bath [52]. Here, different from the Markovian approximation, the approximation is used in the population transition caused by the tunnel coupling $\Delta$. To make this approximation valid, the system should be independent on the past, which means the coherent population change resulted from $\Delta$ should be little. Integrating with Eq. (A.6) we can obtain Eq. (14). 
1. Y. Makhlin, G. Schön, and A. Shnirman, Rev. Mod. Phys. 73, 357 (2001).

2. M.A. Nielsen and I.L. Chuang, Quantum Computation and Quantum Information, Cambridge University Press, Cambridge (2000).

3. J.Q. You and F. Nori, Phys. Today 58, No. 11, 42 (2005).

4. J.E. Mooij, Science 307, 1210 (2005).

5. J. Clarke and F.K. Wilhelm, Nature (London) 453, 1031 (2008).

6. S.N. Shevchenko, S. Ashhab, and F. Nori, Phys. Rep. 492, 1 (2010).

7. A.V. Shytov, D.A. Ivanov, and M.V. Feigel'man, Eur. Phys. J. B 36, 263 (2003).

8. L.D. Landau, Phys. Z. Sowjetunion 2, 46 (1932); G. Zener, Proc. R. Soc. London. Ser. A 137, 696 (1932); E.C.G. Stückelberg, Helv. Phys. Acta 5, 369 (1932).

9. W.D. Oliver, Y. Yu, J.C. Lee, K.K. Berggren, L.S. Levitov, and T.P. Orlando, Science 310, 1653 (2005).

10. M. Sillanpää, T. Lehtinen, A. Paila, Y. Makhlin, and P. Hakonen, Phys. Rev. Lett. 96, 187002 (2006).

11. Y. Nakamura, Y.A. Pashkin, and J.S. Tsai, Phys. Rev. Lett. 87, 246601 (2001).

12. S. Saito, M. Thorwart, H. Tanaka, M. Ueda, H. Nakano, K. Semba, and H. Takayanagi, Phys. Rev. Lett. 93, 037001 (2004).

13. A. Izmalkov, S.H.W. van der Ploeg, S.N. Shevchenko, M. Grajcar, E. Il'ichev, U. Hübner, A.N. Omelyanchouk, and H.-G. Meyer, Phys. Rev. Lett. 101, 017003 (2008).

14. C.M. Wilson, T. Duty, F. Persson, M. Sandberg, G. Johansson, and P. Delsing, Phys. Rev. Lett. 98, 257003 (2007).

15. D.M. Berns, M.S. Rudner, S.O. Valenzuela, K.K. Berggren, W.D. Oliver, L.S. Levitov, and T.P. Orlando, Nature (London) 455, 51 (2008).

16. G.Z. Sun, X.D. Wen, B. Mao, J. Chen, Y. Yu, P.H. Wu, and S.Y. Han, Nature Commun. 1:51 http://dx.doi.org/10.1038/ncomms1050 (2010).

17. J. Bylander, M.S. Rudner, A.V. Shytov, S.O. Valenzuela, D.M. Berns, K.K. Berggren, L.S. Levitov, and W.D. Oliver, Phys. Rev. B 80, 220506 (2009).

18. W.D. Oliver and S.O. Valenzuela, Quant. Inf. Proc. 8, 261 (2009).

19. D.M. Berns, W.D. Oliver, S.O. Valenzuela, A.V. Shytov, K.K. Berggren, L.S. Levitov, and T.P. Orlando, Phys. Rev. Lett. 97, 150520 (2006).

20. M.S. Rudner, A.V. Shytov, L.S. Levitov, D.M. Berns, W.D. Oliver, S.O. Valenzuela, and T.P. Orlando, Phys. Rev. Lett. 101, 190502 (2008).

21. S.O. Valenzuela, W.D. Oliver, D.M. Berns, K.K. Berggren, L.S. Levitov, and T.P. Orlando, Science 314, 1589 (2006).

22. L. Du, Y.Yu, and D. Lan, Fiz. Nizk. Temp. 39, 150 (2013) [Low Temp. Phys. 39, 111 (2013)].

23. I. Goychuk and P. Hänggi, Adv. Phys. 54, 525 (2005).

24. M. Grifoni and P. Hänggi, Phys. Rep. 304, 229 (1998).

25. M.C. Goorden, and F.K. Wilhelm, Phys. Rev. B 68, 012508 (2003).
26. L.J. Du, M.J. Wang, and Y. Yu, Phys. Rev. B 82, 045128 (2010).

27. L.J. Du and Y. Yu, Phys. Rev. B 82, 144524 (2010).

28. L. Du, Y. Yu, and D. Lan, Fiz. Nizk. Temp. 39, 649 (2013) [Low Temp. Phys. 39, No 6 (2013)].

29. S.K. Son, S.Y. Han, and S.I. Chu, Phys. Rev. A 79, 032301 (2009).

30. P. Földi, M.G. Benedict, and F.M. Peeters, Phys. Rev. A 77, 013406 (2008).

31. J. Hausinger and M. Grifoni, Phys. Rev. A 81, 022117 (2010).

32. S. Ashhab, J.R. Johansson, A.M. Zagoskin, and F. Nori, Phys. Rev. A 75, 063414 (2007).

33. Y. Liu, C.P. Sun, and F. Nori, Phys. Rev. A 74, 052321 (2006).

34. C.M. Wilson, G. Johansson, T. Duty, F. Persson, M. Sandberg, and P. Delsing, Phys. Rev. B 81, 024520 (2010).

35. Y.S. Greenberg, Phys. Rev. B 76, 104520 (2007).

36. S.N. Shevchenko, AS. Kiyko, A.N. Omelyanchouk, and W. Krech, Fiz. Nizk. Temp. 31, 752 (2005) [Low Temp. Phys. 31, 569 (2005)].

37. S.N. Shevchenko, S.H.W. van der Ploeg, M. Grajcar, E. Il'ichev, A.N. Omelyanchouk, and H.G. Meyer, Phys. Rev. B 78, 174527 (2008).

38. M. Wubs, K. Saito, S. Kohler, Y. Kayanuma, and P. Hänggi, New J. Phys. 7, 218 (2005).

39. Q. Zhang, P. Hänggi, and J.B. Gong, New J. Phys. 10, 073008 (2008).

40. K. Saito, M. Wubs, S. Kohler, Y. Kayanuma, and P. Hänggi, Phys. Rev. B 75, 214308 (2007).

41. D.T. Pegg, and G.W. Series, Proc. R. Soc. A 332, 281 (1973).

42. J.E. Mooij, T.P. Orlando, L. Levitov, L. Tian, C.H. van der Wal, and S. Lloyd, Science 285, 1036 (1999).

43. I. Chiorescu, Y. Nakamura, C.J.P.M. Harmans, and J.E. Mooij, Science 299, 1869 (2003).

44. M.H.S. Amin, and D.V. Averin, Phys. Rev. Lett. 100, 197001 (2008).

45. M.H.S. Amin and F. Brito, Phys. Rev. B 80, 214302 (2009).

46. D.V. Averin, J.R. Friedman, and J.E. Lukens, Phys. Rev. B 62, 11802 (2000).

47. A. Abragam, The Principles of Nuclear Magnetism, Clarendon, Oxford (1961).

48. R. Rouse, S.Y. Han, and J.E. Lukens, Phys. Rev. Lett. 75, 1614 (1995).

49. J. Johansson, M.H.S. Amin, A.J. Berkley, P. Bunyk, V. Choi, R. Harris, M.W. Johnson, T.M. Lanting, S. Lloyd, and G. Rose, Phys. Rev. B 80, 012507 (2009).

50. D. Goswami, Phys. Rep. 374, 385 (2003).

51. P. Kral, I. Thanopulos, and M. Shapiro, Rev. Mod. Phys. 79, 53 (2007).

52. M.O. Scully, M.S. Zubairy, Quantum Optics, Cambridge University Press, New York (1997). 